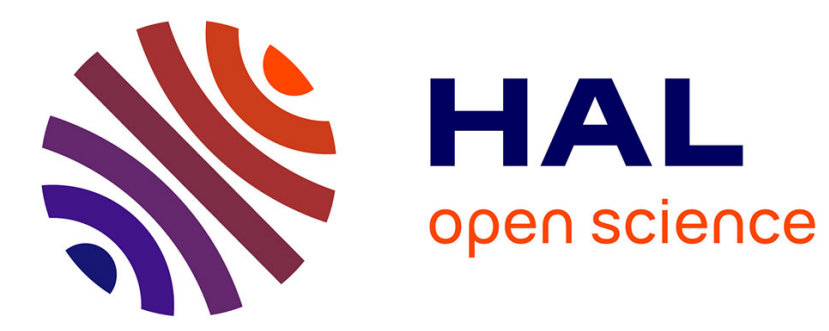

\title{
3D structure and dynamics of filaments in turbulence simulations of WEST diverted plasmas
}

F. Nespoli, P. Tamain, N. Fedorczak, G. Ciraolo, D. Galassi, R. Tatali, E. Serre, Y. Marandet, H. Bufferand, Ph Ghendrih

\section{- To cite this version:}

F. Nespoli, P. Tamain, N. Fedorczak, G. Ciraolo, D. Galassi, et al.. 3D structure and dynamics of filaments in turbulence simulations of WEST diverted plasmas. Nuclear Fusion, 2019. hal-02364554

\section{HAL Id: hal-02364554 https://hal.science/hal-02364554}

Submitted on 15 Nov 2019

HAL is a multi-disciplinary open access archive for the deposit and dissemination of scientific research documents, whether they are published or not. The documents may come from teaching and research institutions in France or abroad, or from public or private research centers.
L'archive ouverte pluridisciplinaire HAL, est destinée au dépôt et à la diffusion de documents scientifiques de niveau recherche, publiés ou non, émanant des établissements d'enseignement et de recherche français ou étrangers, des laboratoires publics ou privés. 


\title{
3D structure and dynamics of filaments in turbulence simulations of WEST
} diverted plasmas

\author{
F. Nespoli1 ${ }^{1,2}$, P. Tamain ${ }^{3}$, N. Fedorczak ${ }^{3}$, G. Ciraolo ${ }^{3}$, D. Galassi ${ }^{2}$, R. Tatali ${ }^{2}$, E. Serre², Y. \\ Marandet $^{1}$, H. Bufferand ${ }^{3}$, Ph. Ghendrih ${ }^{3}$
}

${ }^{1}$ Aix Marseille Univ, CNRS, PIIM UMR 7345, Marseille, France

${ }^{2}$ Aix Marseille Univ, CNRS, Centrale Marseille, M2P2, Marseille, France

${ }^{3}$ IRFM, CEA Cadarache, F-13108 St. Paul-lez-Durance, France

Email: federico.nespoli@univ-amu.fr

\begin{abstract}
We study the effect of a diverted magnetic geometry on edge plasma turbulence, focusing on the three-dimensional structure and dynamics of filaments, also called blobs, in simulations of the WEST tokamak, featuring a primary and secondary X-point. For this purpose, in addition to classical analysis techniques, we apply here a novel fully 3D Blob Recognition And Tracking (BRAT) algorithm, allowing for the first time to resolve the three-dimensional structure and dynamics of the blobs in a turbulent 3D plasma featuring a realistic magnetic geometry. The results are tested against existing theoretical scalings of blob velocity [Myra et al, Physics of Plasmas 2006]. The complementary analysis of the 3D structure of the filaments shows how they disconnect from the divertor plate in the vicinity of the X-points, leading to a transition from a sheath-connected regime to the ideal-interchange one. Furthermore, the numerical results show non-negligible effects of the turbulent background plasma: approximately half of the detected filaments are involved in mutual interactions, eventually resulting in negative radial velocities, and a fraction of the filaments is generated by turbulence directly below the X-point.
\end{abstract}

\section{Introduction}

Turbulence plays a key role in the edge plasma of fusion reactors, determining radial transport in the Scrape-Off-Layer (SOL), driving poloidal rotation and possibly affecting the L-H transition [1]. In the SOL, turbulence takes the form of filamentary structures, usually referred to as blobs [2]. These structures are self-propelled by internal $\mathbf{E} \times \mathbf{B}$ drift radially outwards, i.e. to the first wall. Blobs are therefore believed to increase radial transport in the SOL and, under certain conditions, to accelerate the aging of the first wall through increased erosion [3]. The scaling of blob radial velocity has been therefore subject of several theoretical $[4,5,6,7]$ and experimental $[8,9,10,11,12,13]$ investigations. In particular, it has been shown that blob velocity can be affected by the combined effects of plasma collisionality and the magnetic geometry, leading to multiple regimes for blob dynamics [5] related to different transport properties, depending mainly on whether the filaments are connected to the divertor solid surfaces or not. The transition between different regimes needs thus to be fully understood for both an effective design of the plasma facing components of future fusion reactors and their efficient operation. In a tokamak, the experimental determination of blob size and velocity, i.e. of turbulent radial transport, is subject to several constraints and can be difficult, due mainly to the fact that only a few desired quantities (typically proxies for electron density and plasma potential) are measured typically in 1D (reciprocating Langmuir probes) or at best 2D (fast framing imaging). The 3D structure of blobs remains then unresolved, as it is extremely challenging to couple divertor and midplane measurements and directly determine 
whether the filaments are connected to the plasma sheath at the divertor plates or not.

Numerical simulations of plasma turbulence can help to enhance our understanding of blob structure and dynamics, providing access to a number of physical quantities (not only density and potential, but also parallel current, electron and ion temperatures) at all times, in the whole simulation domain. A number of numerical investigations of blob dynamics has been conducted over the years, ranging from $2 \mathrm{D}$ and $3 \mathrm{D}$ seeded blob simulations (e.g. $[14,15])$ to $3 \mathrm{D}$ simulations in a turbulent background plasma in limited [16] and diverted geometry $[17,18]$. In this work, to improve our understanding of filamentary transport, we analyze in details the three-dimensional structure and dynamics of the blobs in turbulence simulations of plasmas in realistic divertor geometry. Different analysis methods are employed, giving complementary results, namely a Conditional Average Sampling (CAS) technique both in 2D and 3D, and a novel technique for detecting and tracking the blobs directly in 3D, resolving their three-dimensional structure and dynamics, here presented for the first time. In section 2 we introduce the numerical and analysis tools used for this investigation: the turbulence code TOKAM3X [19], the 2D and 3D CAS technique, and the new 3D Blob Recognition And Tracking algorithm (BRAT). The steady-state average profiles of the numerical simulations are described in details in section 3. In section 4 we characterize the average blob dynamics in the poloidal plane. The resulting filament size and radial velocity are compared with the scalings exposed in Refs. [5, 20] in sec. 5. These results are complemented in sec. 6 by the analysis of the three-dimensional structure of the average filaments, allowing us to evaluate the connection of blobs at the outer midplane to the divertor plates. In section 7 we discuss the 3D dynamics of single filaments using the BRAT, and we compare them with the averaged blobs dynamics discussed earlier. Finally, in section 8 the main results are discussed.
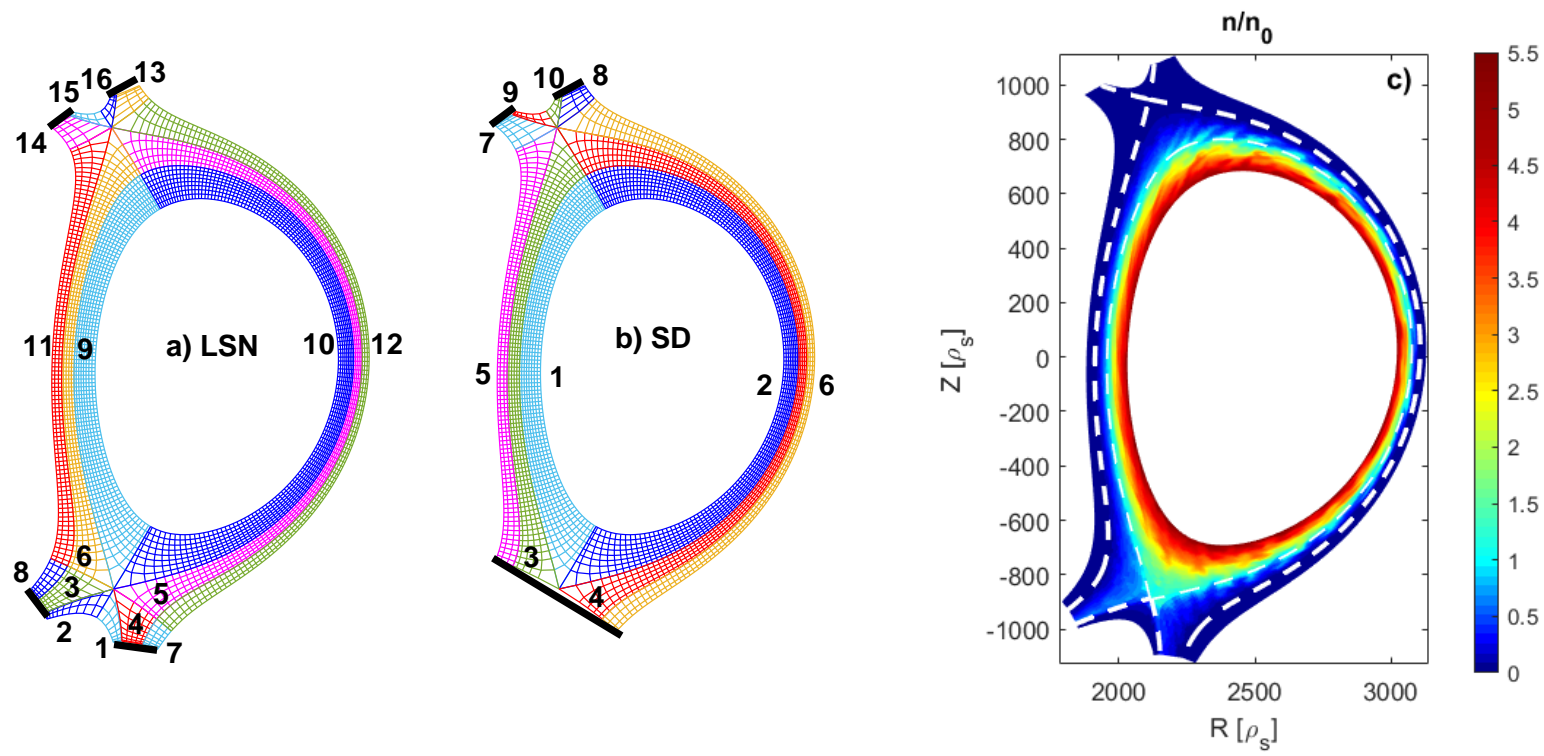

Figure 1: WEST tokamak poloidal plane. Meshes and domain decomposition for (a) lower single null (LSN) and (b) shallow divertor (SD) configurations. The meshes shown here are coarser than the actual meshes used in the simulations. The divertor solid surfaces are shown with thick black lines. (c) Snapshot of the normalized plasma density $n / n_{0}$ in the LSN configuration 


\section{Numerical techniques for the simulations and the analysis}

We consider the edge and SOL plasma of the WEST tokamak [21]. To emphasize the impact of the magnetic geometry on the blobs dynamics, we consider two diverted magnetic configurations, Fig. 1: the lower single null (LSN) and the shallow divertor (SD) magnetic configurations. The LSN configuration (Fig. 1a) is routinely used in WEST, and features a primary X-point close to the target plates and a secondary X-point in the SOL at the top of the machine. The SD configuration (Fig. 1b) corresponds to a limit case, where the $\mathrm{X}$-point is on the target plate.

\subsection{The TOKAM3X code for fluid turbulence simulations}

TOKAM3X is a flux-driven plasma edge turbulence code, evolving self-consistently the background plasma profiles and their fluctuations in versatile and realistic magnetic geometry. Recently, a benchmarking with experimental data from the TORPEX device and with other similar codes has shown some reliable results in the investigation of blobs [22]. In particular, the code solves the continuity equation, the parallel momentum balance equation, electron parallel motion in the form of a generalized Ohms law and the charge conservation equation $(\nabla \cdot j=0)$. The complete model is detailed in Ref. [19]. Neumann conditions are applied at the radial inner and outer boundaries, and Bohm-Chodura boundary conditions are applied at the target plates [23].

A snapshot of the normalized plasma density $n / n_{0}$ for the LSN configuration is shown in Fig. 1c, where $n_{0}=10^{19} \mathrm{~m}^{-3}$ is the reference density used for the normalization of the TOKAM3X equations. We remark that, for this simulation, $n_{0}$ can be considered the density at the Last Closed
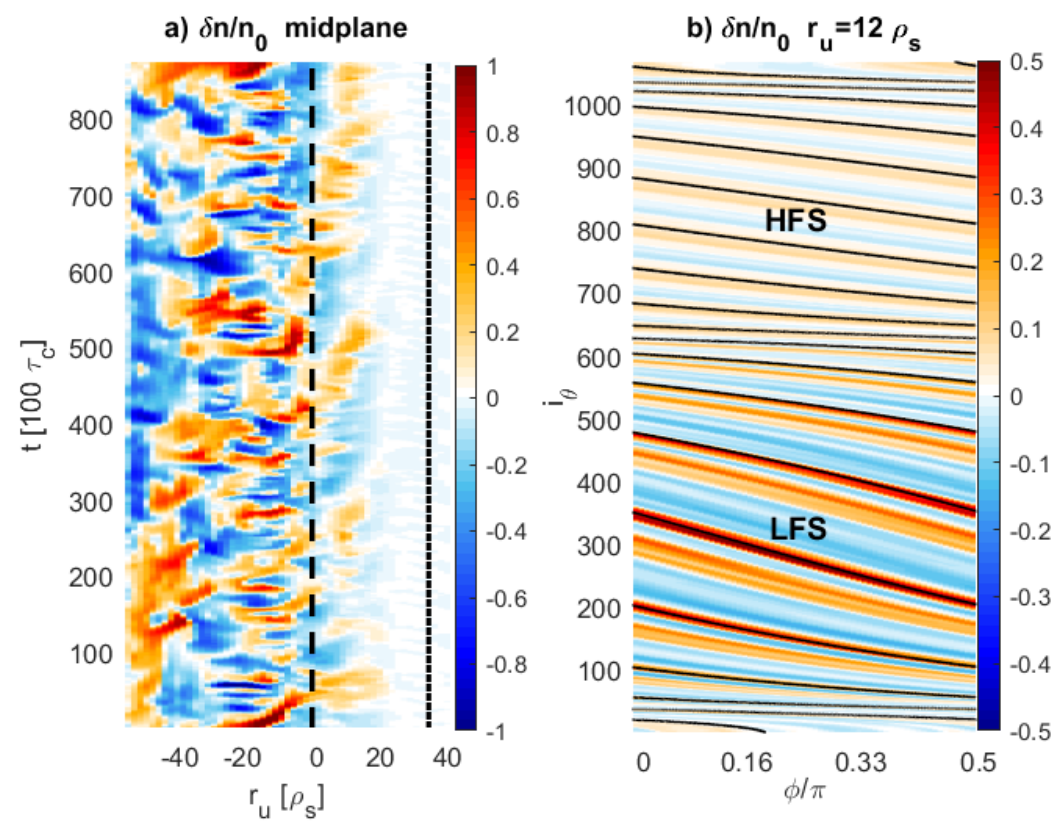

Figure 2: a) Time evolution of the density fluctuation $\delta n$ normalized to the reference density value $n_{0}$, for a radial cut at the OMP. The primary and secondary separatrix are shown with a dashed and dotted black line, respectively. b) Normalized density fluctuation $\delta n / n_{0}$ on a flux surface in the main SOL $\left(r_{u}=12 \rho_{s}\right)$. A magnetic field line resulting from field line tracing is overplotted in black. 
Flux Surface (LCFS), since at this location $n / n_{0} \sim 1$. The plasma is assumed to be isothermal $T_{e}=T_{i}=T_{0}$, with $T_{0}=50 \mathrm{eV}$ the electron temperature at the LCFS. This allows us a more accurate comparison with the scalings derived in Ref. [5], by avoiding secondary effects due to finite temperature [24] and neutral friction [25].

The simulation includes a realistic safety factor $q$ varying poloidally, with its value just inside the LCFS being $q_{95} \sim 4$. The effects of a finite aspect ratio $A=R_{0} / a=5$ are included, where $R_{0}$ and $a$ are the major and minor radius, respectively. To ensure a tractable computational time together with a reliable resolution, the computational domain is approximately $1 / 3$ of the real size of WEST, with $1 / \rho *=a / \rho_{s}=512, \rho_{s}$ being the ion sound speed Larmor radius, and a $\pi / 2$ periodicity of the solution is assumed to consider only $1 / 4$ of torus. In addition, the plasma collisionality is set to $\nu^{*} \equiv \rho_{s} /\left(c_{s} \tau_{e}\right)=0.072$, where $\tau_{e}$ is the electron collision time. The choice of this value, approximately a factor of 5 larger than the typical experimental one, is driven by the need to keep computational costs reasonable [23], since small collisionality leads to smaller turbulent structures and requires grid with higher resolution. The impact of this parameter on blob dynamics will be further discussed in section 5 .

The meshes used for the two configurations are sketched in Fig. 1a,b. It is regular in the toroidal direction with $N_{\phi}=64$ poloidal planes. In the poloidal plane, the domain decomposition allows us to consider variable resolutions in the poloidal $(\theta)$ and radial $(\psi)$ directions depending on the different zones (16 for the LSN configuration). In a current run, the mesh size is approximately of $6.4 \cdot 10^{4}$ grid points in the poloidal plane. The output sampling frequency is $1 / 100 \tau_{c}^{-1}$, where $\tau_{c}$ is the ion cyclotron time. In summary, our simulations feature a realistic magnetic geometry. The size of the simulation $(1 / \rho *)$ and the grid resolution, together with the collisionality $\nu^{*}$, are chosen as a trade-off between containing computational cost while maintaining a high enough resolution to allow turbulence to develop in the simulations and to be studied afterwards. This is indeed the case, as it emerges from Fig. 2a, where an example of the evolution in time of the density fluctuation $\delta n$ at the outer midplane (OMP) is shown for the LSN configuration. The picture shows that in these simulations turbulent structures arise spontaneously inside the LCFS (black dashed line) and extend into the SOL, where they propagate radially outwards. These turbulent structures are elongated in the toroidal direction, as is visible in Fig. $2 \mathrm{~b}$, where $\delta n$ is shown on a flux surface in the main SOL (region comprised between the two separatrixes). In the picture, a magnetic field line resulting from field line tracing is overplotted in black, showing that the density fluctuations are field aligned.

\section{$2.22 \mathrm{D}$ and 3D Conditional Average Sampling (CAS)}

To investigate blob dynamics we use a Conditional Average Sampling (CAS) technique, as is commonly done in experiments using Langmuir probes arrays or fast imaging. In experiments, due to obvious constraints on the diagnostics position, this analysis is usually limited to a very specific portion of space, typically restricted to a line or at best to a narrow $2 \mathrm{D}$ region usually at the outer midplane. In 3D simulations, this constraint is no longer present and each point of the $3 \mathrm{D}$ domain is accessible for CAS analysis. At a fixed point $\mathbf{x}_{\mathbf{0}}=\left(\psi_{0}, \theta_{0}, \phi_{0}\right)$ in the simulation domain, the blobs are identified by the threshold condition $n\left(\mathbf{x}_{\mathbf{0}}, t\right)>\bar{n}\left(\mathbf{x}_{\mathbf{0}}\right)+2.5 \sigma\left(\mathbf{x}_{\mathbf{0}}\right)$, where $\bar{n}$ is the time and toroidally averaged density and $\sigma$ is the corresponding standard deviation. The peaks of the density time trace $n\left(\mathbf{x}_{\mathbf{0}}, t\right)$ for which the condition is satisfied define the time of occurrence of a blob, $t_{k}$. The conditional averaged blob spatial profiles are computed superimposing the density fluctuation profiles at the corresponding peak times $\delta n_{C A S}(\mathbf{x})=\sum_{k=1}^{N}\left(n\left(\mathbf{x}, t_{k}\right)-\bar{n}(\mathbf{x})\right) / N$. The conditional average radial and poloidal size of the blob, $a_{r}$ and $a_{\theta}$ respectively, are computed as the HWHM of $\delta n_{C A S}(\mathbf{x})$ in the $r$ and $\theta$ direction respectively, with an incertitude of $\pm 2 \rho_{s}$. The blob 
velocity is identified with the $\mathbf{E} \times \mathbf{B}$ drift velocity, averaged over the blob occurrence times $t_{k}$, whose three components can be readily computed from the plasma potential at any given time and spatial position in the simulation domain. As the reference point $\mathbf{x}_{\mathbf{0}}$ is swept across the whole simulation domain, we can compute the desired CAS quantities in 3D. Though, all the results are averaged on the toroidal coordinate to improve statistics, resulting in 2D maps on a poloidal plane. Points in space where the statistic is too scarce and /or the background averaged density is too small $\bar{n}<n_{0} / 100$, where $n_{0}$ is approximately the density at the LCFS, are excluded from the following analysis.

Furthermore, we use a 3D CAS technique to evaluate the three-dimensional structure of the average blobs in the main SOL. Similarly to the 2D CAS technique exposed above, we use the time trace of the density in a reference point $\mathbf{x}_{\mathbf{0}}=\left(\psi_{0}, \theta_{0}, \phi_{0}\right)$ as a trigger, identifying the blobs through the threshold condition $n\left(\mathbf{x}_{\mathbf{0}}, t\right)>\bar{n}\left(\mathbf{x}_{\mathbf{0}}\right)+2.5 \sigma\left(\mathbf{x}_{\mathbf{0}}\right)$. For a generic field $f$ we then compute the CAS field in the whole simulation domain $\mathbf{x}=(\psi, \theta, \phi)$ as $f_{C A S, \mathbf{x}_{\mathbf{0}}}(\mathbf{x})=\sum_{k=1}^{N} f\left(\mathbf{x}, t_{k}\right) / N$, where $t_{k}$ are the times at which the blobs are detected at the point $\mathbf{x}_{\mathbf{0}}$. This procedure is repeated for all the available poloidal planes $\phi_{0}$ to increase statistics. We restrict this study to the blobs in the main SOL at the OMP, computing the CAS of density $n$, electric potential $\Phi$, parallel current $j_{\|}$and radial $\mathbf{E} \times \mathbf{B}$ velocity $v_{r}$ and the respective fluctuations $\delta n, \delta \Phi, \delta j_{\|}$.

\subsection{A new 3D blob recognition and tracking algorithm (BRAT)}

Blob tracking techniques have been used on both experimental data (Langmuir probe arrays [8] and gas puff imaging [26]) and simulations [16]. These techniques are usually implemented in 2D only, since the experimental measurements are available in a single poloidal plane, providing the blob poloidal section and velocity on the chosen poloidal plane. In $3 \mathrm{D}$ turbulent simulations, the constraint of a single poloidal plane can be dropped, and the detection and tracking of the blob can be implemented directly in 3D leading to the time evolution of the whole spatial structure of the filaments.

A new Blob Recognition And Tracking algorithm (3DBRAT) has been developed in this work . For every timestep of the simulation $t_{k}$, the blob recognition is performed over the whole simulation domain through the condition

$$
n\left(\mathbf{x}, t_{k}\right)>\bar{n}(\mathbf{x})+\Theta_{d} \sigma(\mathbf{x}),
$$

where $\Theta_{d}$ is the detection threshold, $\mathbf{x}=(\psi, \theta, \phi)$ is the position vector, and the bar denotes averaging over time and toroidal direction. The connected domains satisfying this condition are identified and labeled with integer values $\left\{L_{i}\right\}_{i=1, \ldots, N_{b}}$. We remark that no assumption on the field alignment of the structures is made during this detection procedure. Though, the blobs are typically elongated along the field lines and extend toroidally through multiple tours of the torus. The simulation domain, though, covers at best a toroidal extent of $2 \pi$, and periodic boundary conditions are applied at the toroidal borders, $i_{\phi}=1$ and $i_{\phi}=N_{\phi}$. Therefore, a single blob would be originally detected as multiple connected domains extending from one toroidal border to the other, with different labels $L_{i}$. The connected domains extending through the toroidal borders need to be identified and to be given the same label. In TOKAM3X simulations there is an additional technical issue related to the simulation domain splitting (see on Fig. 1), in which a single blob can extend through multiple zones. The patching of the blobs through different zones and in the toroidal direction is performed simultaneously. The blob toroidal starting point is identified, so that the different toroidal sections of the same filament can be associated with a progressively increasing "tour number" $N_{\text {tour }}$, and a monotonically increasing extended toroidal coordinate $\phi_{\text {ext }}=\phi+N_{\text {tour }} \phi_{\max }$, no longer restricted to the interval $[0,2 \pi)$, where $\phi_{\max }=\phi\left(i_{\phi}=N_{\phi}\right)$. In this way, each blob will intersect the poloidal 


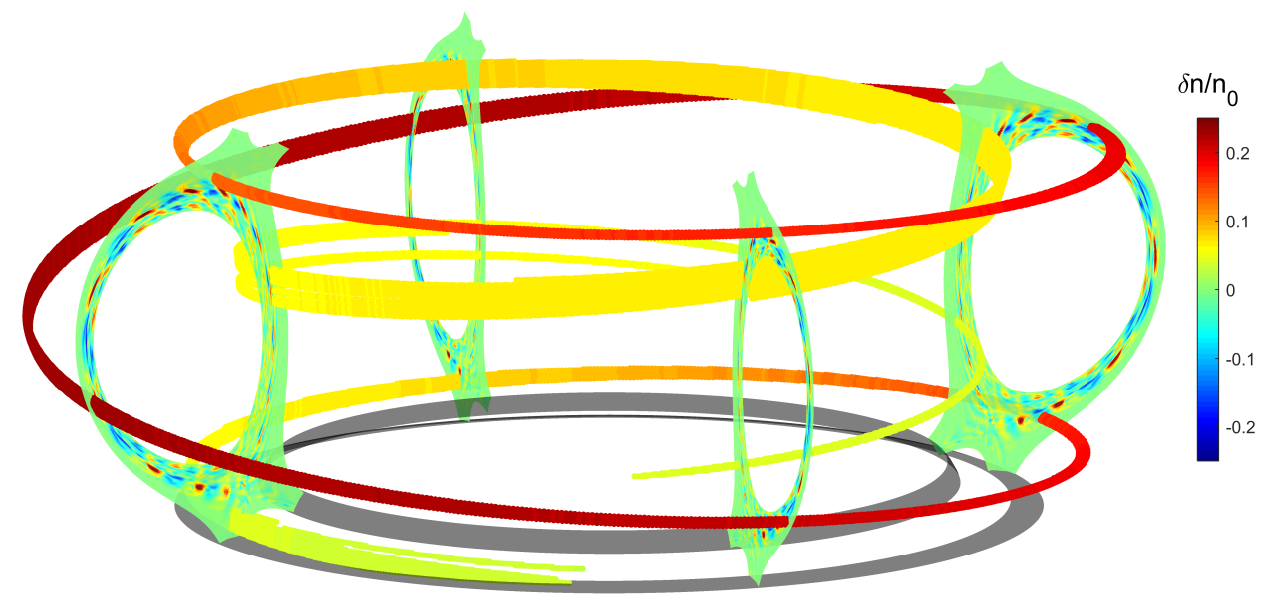

Figure 3: Three-dimensional structure of one blob in the main SOL as resulting from the 3DBRAT, color coded with the average density fluctuation for each poloidal plane. The density fluctuation field on four different poloidal planes is also shown. The lower divertor plates are shown with black surfaces.

plane identified by $\phi=\phi_{\text {ext }}$ only once, allowing for the computation of the blob center of mass and velocity, as it will be clarified in the following. Indeed, for every poloidal plane of the "unraveled" blob $\phi=\phi_{\text {ext }, i}$, we compute the position of the center of mass as $\mathbf{x}_{C M, i}=\sum \delta n V \mathbf{x} / \sum \delta n V$, where the sum is over all the simulation cells satisfying Eq. 1 on the $\phi=\phi_{\text {ext, } i}$ plane and $V$ is the volume of each cell. Similarly, we define for each poloidal plane an average density fluctuation $\delta n_{C M, i}=\sum \delta n V / \sum V$. This allows us to define a toroidal center of mass for the blob $\phi_{C M}=\sum \delta n_{C M, i} V_{i} \phi_{\text {ext }, i} / \sum \delta n_{C M, i} V_{i}$, where $V_{i}$ is the total volume of the blob in the $\phi=\phi_{\text {ext }, i}$ poloidal plane. Also, for each $\phi=\phi_{\text {ext }, i}$ plane, the poloidal and radial size of the blob are computed as HWHM of the density fluctuation in the poloidal and radial direction, respectively.

For a better calculation of center of mass and velocity, we allow for two different threshold for the blob detection, the first one defining the blob shape that finally impacts the computation of the center of mass and velocity of the blob, and a second one that sets the definition of what we consider a blob, similarly to the CAS analysis. Therefore, of all the detected blobs at the time $t_{k}$, only the ones for which at least one point satisfies $n\left(\mathbf{x}, t_{k}\right)>\bar{n}(\mathbf{x})+\Theta_{b} \sigma(\mathbf{x})$ are kept for the following analysis, where $\Theta_{b} \geq \Theta_{d}$ is the threshold defining the blobs; in the following analysis, we will use $\Theta_{d}=1.5$ and $\Theta_{b}=2.5$. An example of the structure of a blob resulting form this detection technique is shown in Fig. 3, color coded with the average density fluctuation $\delta n_{C M, i}$ for each poloidal plane.

In order to track the filaments in time, the blob $b_{i}$ detected at the time $t_{k}$ have to be identified as the evolution of the blob $b_{j}$ detected at the previous time step $t_{k-1}$. This is done through a superposition criteria: the two blobs are considered to be the same if the volume of their superposition is bigger than a fraction $f_{\text {sup }}=0.1$ of the volume of the former structure, $V\left(b_{i}\left(t_{k}\right) \cap b_{j}\left(t_{k-1}\right)\right)>f_{\text {sup }} V\left(b_{j}\left(t_{k-1}\right)\right)$. Using this criteria, the algorithm is able to recognize merging and splitting events. Merging occurs when two or more distinct blobs at time $t_{k-1}$ superimpose with one blob at time $t_{k}$, while splitting occurs when one blob at time $t_{k-1}$ superimposes with two 

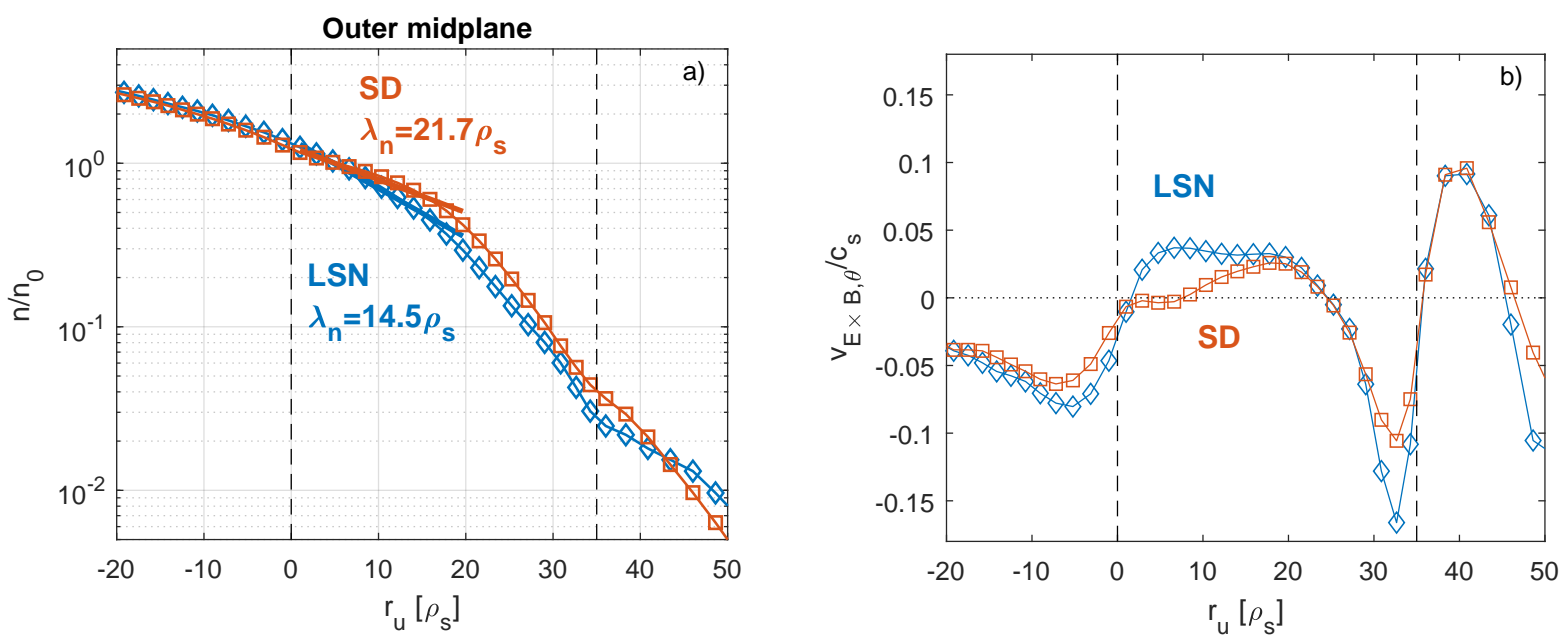

Figure 4: Radial profiles at the outer midplane of a) density $n$ b) poloidal velocity $v_{E \times B, \theta}$ for the lower single null (blue diamonds) and shallow divertor (orange squares) configurations. The primary and secondary separatrixes are shown with black dashed lines. In a), the fit of the $n\left(r_{u}\right)$ profiles in the SOL with an exponential up to $r_{u}=0$ is shown with thick solid lines.

or more distinct blobs at time $t_{k}$; combined splitting and merging of several blobs into several blobs is possible and is also taken into account.

Once the blobs are tracked in time, we compute their velocity, defined for every poloidal plane $\phi=\phi_{e x t, i}$ as the velocity of the center of mass $\mathbf{v}_{C M, i}\left(t_{k}\right)=\left(\mathbf{x}_{C M, i}\left(t_{k+1}\right)-\mathbf{x}_{C M, i}\left(t_{k-1}\right)\right) / 2 \mathrm{~d} t$ and the angular velocity of the blob as $\omega_{C M}=\left(\phi_{C M}\left(t_{k+1}\right)-\phi_{C M}\left(t_{k-1}\right)\right) / 2 \mathrm{~d} t$. All the blobs living less then three time steps are discarded from the analysis since the computation of the velocity would not be possible.

\section{Plasma steady state.}

We here characterize the plasma steady-state in both LSN and SD magnetic configurations using time-averaged profiles.

The density radial profiles $n\left(r_{u}\right)$ at the outer midplane, with $r_{u}$ the upstream radial coordinate being $r_{u}=0$ at the LCFS, clearly show the effect of the secondary separatrix (i.e. the most radially outwards one) for both configurations, Fig. 4a. Indeed, the profiles exhibit in the SOL two different slopes in the vicinity of the two separatrixes, with a steepening of the profiles as one moves towards the secondary one. This increases of gradients can be due to the presence of a shear layer of the poloidal $\mathbf{E} \times \mathbf{B}$ velocity for $r_{u} \gtrsim 20 \rho_{s}$, as shown in Fig. $4 \mathrm{~b}$, where the radial profiles of $v_{\theta}$ are plotted. Similarly to what observed in ASDEX-Upgrade [27], this shear layer can be caused by the sudden reduction in connection length when crossing the secondary separatrix. The SOL decay length is computed fitting the $n\left(r_{u}\right)$ profiles from the LCFS up to $r_{u}=20 \rho_{s}$ where the change in slope appears. For the LSN and SD configurations, the values are $\lambda_{n}=14.5 \rho_{s}$ and $\lambda_{n}=21.7 \rho_{s}$, respectively. The widening of the SOL for the SD configuration, reflected in a decrease of the poloidal shear at the LCFS, is unexpected and requires further investigations.

For both the LSN and SD configurations, the presence of the secondary separatrix affects the profiles at the target plates as well, as shown on Fig. 5 showing the profiles of the particle flux $\Gamma\left(r_{u}\right)$ along the upstream radial coordinate. The profiles are fitted with the convolution of an exponential decay 

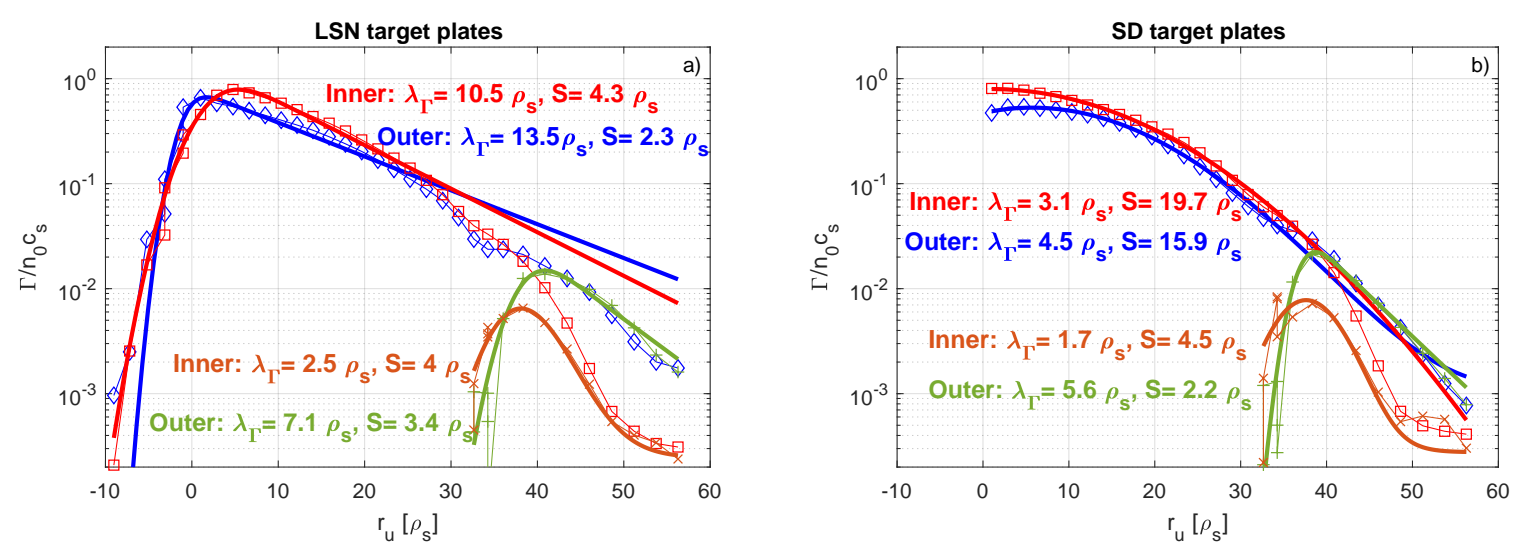

Figure 5: Radial profiles remapped upstream of the particle flux at the target plates $\Gamma$ for a) the LSN case b) the SD case. The main targets are shown with red squares and blue diamonds for the HFS and LFS respectively. The secondary targets are shown with orange $\mathrm{X}_{\mathrm{s}}$ and green crosses for the HFS and LFS respectively. The fit of the $\Gamma\left(r_{u}\right)$ profiles with Eq.(2) are shown with solid lines.

with a Gaussian of width S, describing a spreading of the profile due to diffusion in the divertor $[28,29]$ :

$$
f(x)=\frac{f_{0}}{2} \exp \left[\left(\frac{S}{2 \lambda}\right)^{2}-\frac{x-x_{0}}{\lambda_{i}}\right] \operatorname{erfc}\left(\frac{S}{2 \lambda}-\frac{x-x_{0}}{S}\right)
$$

For both LSN and SD configurations, the profiles at the main (lower) divertor are satisfactorily described by Eq.(2) up to $r_{u} \sim 30 \rho_{s}$, while this is no longer true as one approaches and crosses the secondary separatrix. For the LSN configuration, the fitted values of the decay length $\lambda$ is comparable with the ones computed upstream for the density profiles, the HFS SOL being slightly narrower than the LFS SOL, consistently with the ballooning character of the turbulent transport. For the SD configuration, the fit with Eq.(2) provides values of the spreading parameters much bigger than the exponential decay length $S \gg \lambda$. This is due partly to the lack of a private flux region, allowing to correctly estimate $S$, and partly to the fact that the profiles are actually more Gaussian-like. Eq. (2) might therefore no longer provide a meaningful physical description of the target profiles. The presence of the secondary X-point leads to the deposition of particles on the upper divertor plates $(\Gamma>0)$, even though the peak value is more than one order of magnitude smaller than the one at the main divertor. The $\Gamma\left(r_{u}\right)$ profiles at the secondary targets are in general well described by Eq.(2), and they are dominated by diffusion $(S \geq \lambda)$.

Finally, in both LSN and SD configurations, we observe an inner/outer asymmetry, with the ratii of the peak values $\Gamma_{\text {peak,inner }} / \Gamma_{\text {peak,outer }} \sim 1.2$ and 1.5 , respectively. This could be partially due to the presence of a radial $\mathbf{E} \times \mathbf{B}$ drift, directed across the X-point towards the HFS, similarly to what previously observed in TOKAM3X simulations in diverted geometry [30]. Also, this inner/outer asymmetry is reversed at the secondary target that is consistent with the LFS secondary target, and this is consistent with the LFS secondary target being directly connected to the turbulent LFS SOL, while the HFS secondary target is connected to the less turbulent HFS SOL. Furthermore, the radial $\mathbf{E} \times \mathbf{B}$ through the secondary $\mathrm{X}$-point is reversed with respect to the primary X-point, directed here towards the LFS. 

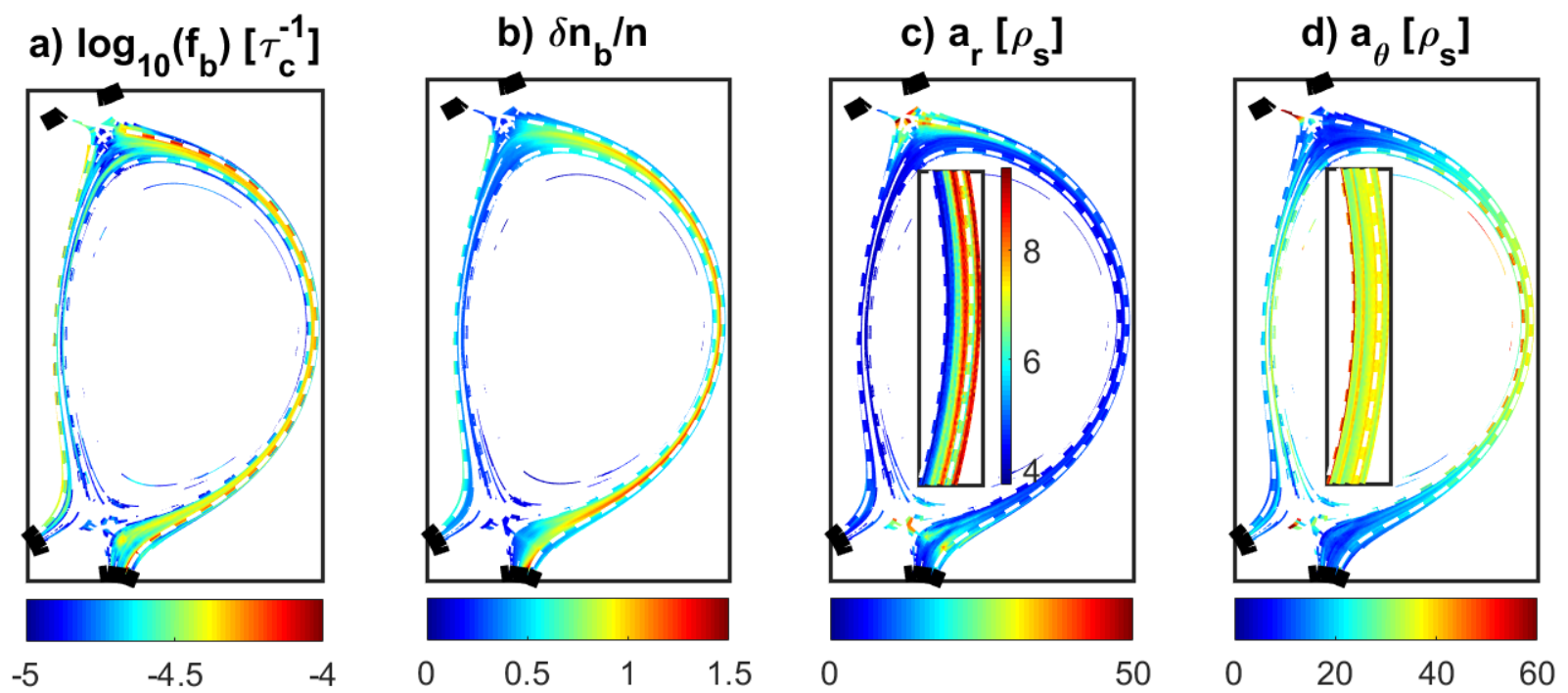

Figure 6: Poloidal distribution of the CAS results for the LSN case, respectively a) the blob detection frequency $f_{b}$ b) the blob relative density fluctuation $\delta n_{b} / n$ c) blob radial size $a_{r}$ and d) blob poloidal size $a_{\theta}$. For pictures c) and d), a zoom of the region around the OMP is also shown; note the different color coding for the subplot in c).
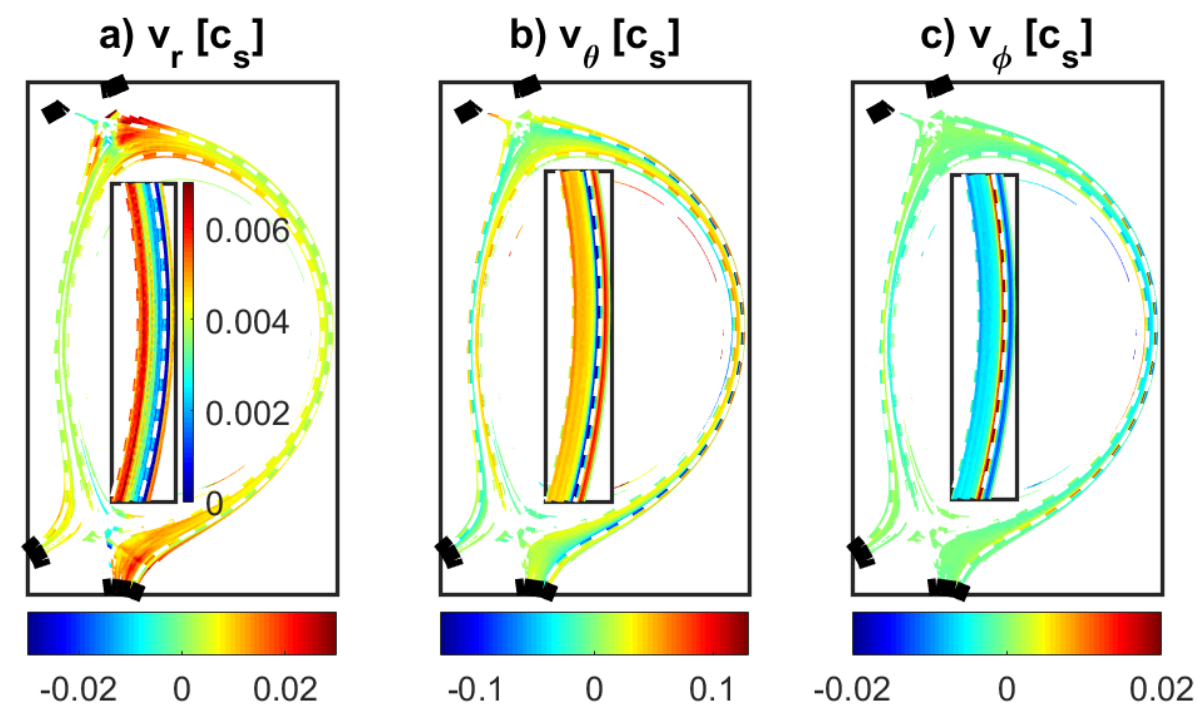

Figure 7: Poloidal distribution of the blob velocities resulting from the CAS for the LSN case, respectively a) radial b) poloidal and c) toroidal component. For all quantities, a zoom of the region around the OMP is also shown; note the different color coding for the subplot in a).

\section{Blob dynamics in the poloidal plane}

The blob dynamics is analyzed here in both LSN and SD magnetic configurations using the 2D conditional average sampling technique (2D CAS) described in section 2.2 . 
Fig.6a shows the blob detection frequency $f_{b}$ for the LSN configuration. As expected, blobs occur more frequently in the main LFS SOL, as is expected since this region features unfavorable curvature, developing interchange instability. The maximum of the relative density fluctuation of the blobs $\delta n_{b} / n$ is also peaked in the LFS SOL as shown in Fig.6b due to the ballooning character of transport. Both $f_{b}$ and $\delta n_{b} / n$ decrease strongly in the vicinity of the primary X-point, exhibiting a quiescent zone, similarly to what observed experimentally in the MAST tokamak with fast framing imaging [31] and in previous TOKAM3X simulations in COMPASS geometry [30].

At the OMP, the radial size of the blobs $a_{r}$ is in the range $4 \leq a_{r} / \rho_{s} \leq 10$ (inner plot in Fig. 6c), increasing moving radially outwards. $a_{r}$ is reduced close to the secondary separatrix consistently with the shear in the poloidal velocity at this location, as previously discussed in section 3 (Fig. 4). Moving poloidally towards the X-points, the blob radial size increases up to a factor 5 with respect to its value at the OMP, accordingly to the flux expansion. The blob poloidal size $a_{\theta}$ (Fig. 6d) exhibits an opposite behaviour, with $29 \leq a_{\theta} / \rho_{s} \leq 59$ at the OMP and local maxima close to the two separatrixes, consistently with the shearing of the poloidal velocity. $a_{\theta}$ decreases by approximately a factor 5 approaching the X-points, consistently with the conservation of flux along the field lines and the above mentioned increase in the radial size.

The three components of blob velocities resulting from the CAS are showed in Fig. 7. The radial velocity $v_{r}$ is usually positive, assuming slightly negative values only in the vicinity of the $\mathrm{X}$-points, accordingly to the steady-state $\mathbf{E} \times \mathbf{B}$ flow discussed in the previous section. At the OMP, the blob motion is mainly in the poloidal direction, being $v_{\theta} / v_{r} \sim 10$ and $v_{\theta} / v_{\phi} \sim 10$. Here, $v_{r}$ decreases moving radially outwards while $v_{\theta}$ changes sign close to the secondary separatrix, accordingly to the steady-state $\mathbf{E} \times \mathbf{B}$ flow. This change of direction in blob propagation is observed also in the toroidal direction. As one approaches the X-points, $v_{r}$ increases by approximately 5 times, accordingly to the flux expansion and the hypothesis of the filaments being field-aligned.

Results for the SD magnetic configuration are qualitatively the same, though some differences emerge. The blob detection frequency increases by approximately $50 \%$ with respect to the LSN configuration, with an average value of $\left\langle f_{b}\right\rangle=3.9 \cdot 10^{-5} \tau_{c}^{-1}$ compared to $\left\langle f_{b}\right\rangle=2.6 \cdot 10^{-5} \tau_{c}^{-1}$ for the LSN. The blob size also increases both radially and poloidally with respect to the LSN configuration, with $5 \leq a_{r} / \rho_{s} \leq 12$ and $34 \leq a_{\theta} / \rho_{s} \leq 56$ at the OMP. In the vicinity of the LCFS at the OMP, the blob radial velocity is increased by $\sim 25 \%$ with respect to the LSN configuration, while both the poloidal and toroidal velocity decrease by $\sim 50 \%$.

The poloidal variations of the blob radial velocity $v_{r}$ on a flux surface is shown on Fig. 8a,b for both the LSN and SD configuration respectively. $v_{r}$ increases when approaching both the primary and the secondary X-point. This is due to the fact that the filamentary structures are field-aligned, and as they move across flux surfaces at the OMP, they remain aligned with the same flux surface at the divertor entrance as well. For this to happen, the filaments have to increase their radial velocity proportionally to the flux expansion $f_{x}$, since the distance between flux surfaces increases progressively when moving from the OMP to the X-points. This is clearly shown on Fig. 9, where the blob radial velocity normalized to the flux expansion $v_{r} / f_{x}$ is plotted along the flux surface for the two divertor configurations. The $v_{r} / f_{x}$ profiles for each flux surface are approximately flat both at the LFS and HFS, consistently with the previous explanation. The sudden decrease in $v_{r}$ when moving from the LFS to the HFS $\left(s / L_{\theta} \sim 0.6\right)$ suggests that the filamentary structures at the HFS are disconnected from the ones at the LFS. 

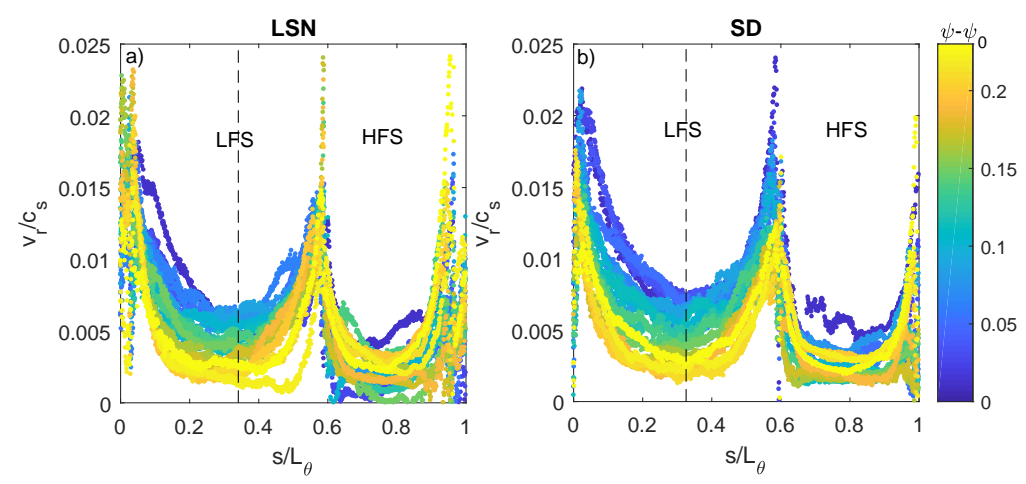

Figure 8: Blob radial velocity $v_{r}$ variations at different $\Psi$ resulting from the CAS plotted along each flux surface in the main SOL versus the normalized poloidal coordinate $s / L_{\theta}=0$ at the LFS target and $s / L_{\theta}=1$ at the HFS target, for a) LSN and b) SD configuration. The position of the OMP is shown with a black dashed line.
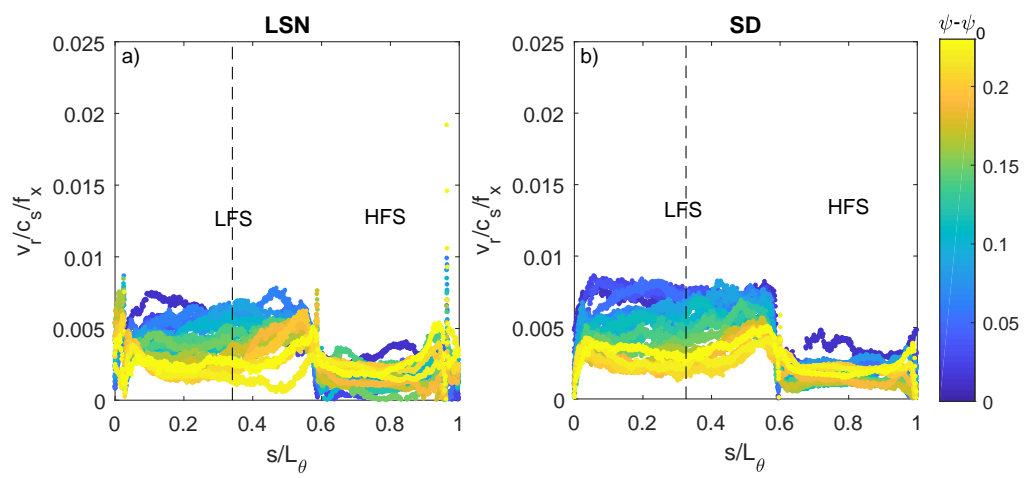

Figure 9: Blob radial velocity $v_{r}$ resulting from the CAS, normalized by the flux expansion $f_{x}$ and plotted along each flux surface in the main SOL along the normalized poloidal coordinate $s / L_{\theta}=0$ at the LFS target and $s / L_{\theta}=1$ at the HFS target, for a) LSN and b) SD configuration. The position of the OMP is shown with a black dashed line.

\section{Comparison with theoretical scalings}

The radial velocity and poloidal sizes of blobs resulting from the 2D CAS are now compared to the scaling laws derived in Ref. [5]. This model is particularly well-suited here for comparisons since the plasma is assumed to be isothermal. The density and electric potential fluctuations along a flux tube are considered, as it extends along the magnetic field with varying fanning and shear. As a result of this derivation, the normalized blob velocity $\tilde{v}=v_{r} / v^{*}$ at the outer midplane (OMP) scales differently with the normalized blob size $\tilde{a}=a_{\theta} / a^{*}$ depending on the adimensional parameters $\Lambda=\nu^{*}\left(m_{e} / m_{i}\right)\left(L_{\|} / \rho_{s}\right), \epsilon_{x}=1 / f_{x, \max }$ and $\Theta=\tilde{a}^{5 / 2}$, where $L_{\|}$is the connection length from the OMP to the X-point, $v^{*}=\left(L_{\|} \rho_{s}^{2} / R_{0}^{3}\right)^{1 / 5} c_{s}$ and $a^{*}=\left(L_{\|}^{2} / R_{0} \rho_{s}\right)^{1 / 5} \rho_{s}$ are the typical blob radial velocity and size, respectively. $f_{x, \max }$ is, for each flux surface, the maximum flux expansion along the flux tube, corresponding approximately to the flux expansion at the level of the X-point.

The main instability drive, the curvature driven interchange modes around the outer midplane, can be balanced by different mechanisms, leading to four different regimes, namely:

- sheath connected (CS), typical of low collisionality plasmas, where the instability drive is 

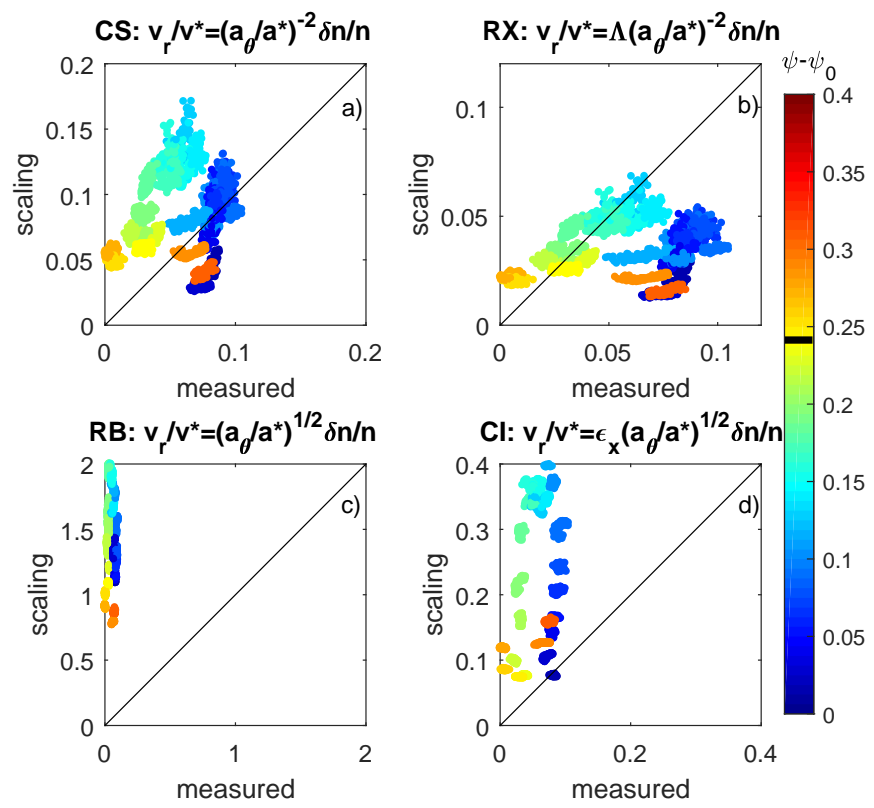

Figure 10: Comparison between the normalized blob radial velocity $\tilde{v}=v_{r} / v^{*}$ measured with the 2D CAS technique with the prediction of the four different scalings: a) sheath connected (CS) b) resitive X-point $(\mathrm{RX}) \mathrm{c}$ ) resistive ballooning (BR), and d) connected ideal-interchange (CI), for the LSN configuration, color coded with the distance from the LCFS in term of poloidal magnetic flux $\psi$, the black line on the colorbar indicating the position of the secondary separatrix.
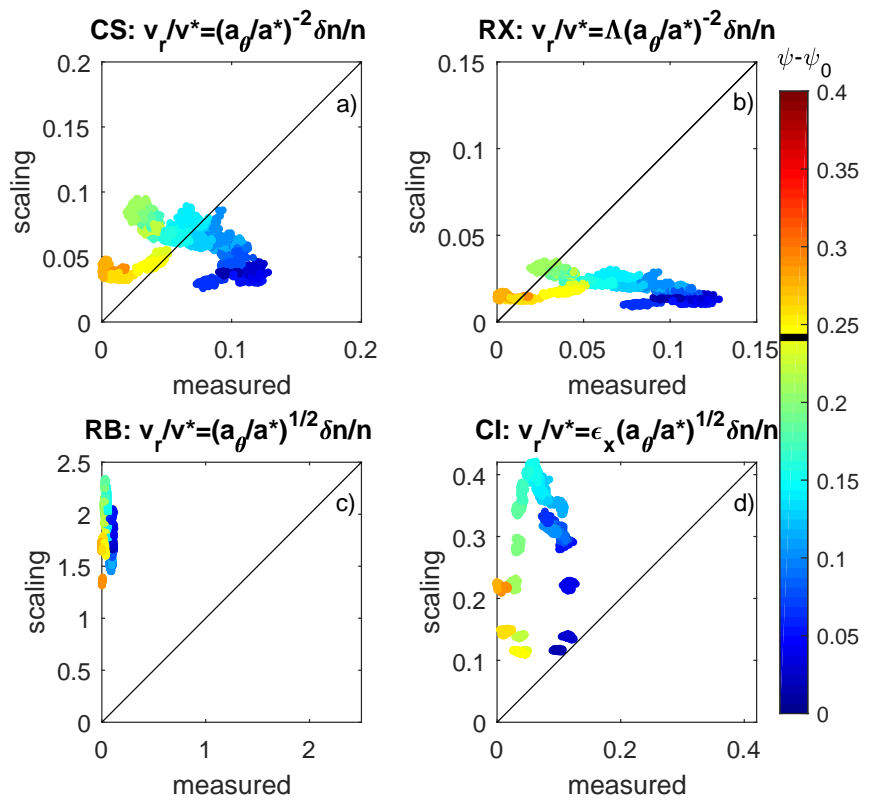

Figure 11: Comparison between the normalized blob radial velocity $\tilde{v}=v_{r} / v^{*}$ measured with the 2D CAS technique with the prediction of the four different scalings: a) sheath connected (CS) b) resitive X-point $(\mathrm{RX})$ c) resistive ballooning (BR), and d) connected ideal-interchange (CI), for the SD configuration, color coded with the distance from the LCFS in term of poloidal magnetic flux $\psi$, the black line on the colorbar indicating the position of the secondary separatrix. 
balanced by sheath-connected modes. The blob velocity scales as $\tilde{v}=1 / \tilde{a}^{2}$.

- connected ideal-interchange (CI), where the instability drive is balanced by fanning-enhanced inertia in a second region away from the OMP (typically an X-point). The blob velocity scales as $\tilde{v}=\epsilon_{x} \tilde{a}^{1 / 2}$

- resistive X-point (RX), balancing the instability drive with parallel resistivity, blob velocity scales as $\tilde{v}=\Lambda / \tilde{a}^{2}$

- resistive ballooning $(\mathrm{RB})$, balancing the instability drive with inertia around the OMP, blob velocity scales as $\tilde{v}=\tilde{a}^{1 / 2}$

Investigations in Ref. [20,32] have shown that the background plasma impacts the blob radial velocity proportionally to the relative pressure fluctuations. So, all the above scalings should be multiplied by $\delta n_{b} / n$, since in the present isothermal simulations the pressure can be identified with the density. We remark that, even though the value of collisionality $\nu^{*}$ is artificially high in our simulations, this does not force the blobs to be in the RB or RX regimes. Indeed the parameter controlling the transition between the different regimes are $\Lambda=\nu^{*}\left(m_{e} / m_{i}\right)\left(L_{\|} / \rho_{s}\right)$ and $\epsilon_{x}=1 / f_{x, \max }$. For our simulations, $\Lambda \lesssim 0.5$, so we expect the blobs to be in the CS regime (transition between CS and $\mathrm{RX}$ or RB for $\Lambda \sim 1$ ), or in the CI one (transition between CS and CI or RX for $\Theta \sim 1 / \epsilon_{x} \lesssim 16$ ).

The blob sizes and velocities resulting from the 2D CAS at the LFS (an angular sector of $\pm \pi / 3$ around the OMP) are compared with the four different scalings in Fig. 10 and 11 for the LSN and SD configurations, respectively, color coded with the distance of the flux surfaces from the LCFS. For all cases, the equality between the measured radial velocities and the ones predicted by the scaling laws is shown with a black line.

For the LSN configuration, numerical results in the main SOL (in blue in the figure) agree well with the CS scaling (a) (blobs connected to the divertor plates), while moving further outwards in the SOL (light blue, green, yellow points), the agreement becomes better with the RX scaling (b), though the two only differ by a factor $\Lambda$, that in this case is close to unity. As one moves inwards towards the LCFS, the squeezing and fanning of the flux tubes, due to the flux expansion and magnetic shear, become important, and the blobs velocity are better described by the CI scaling (d). The latter case corresponds to the dark blue points in the figure.

Qualitatively, the same conclusions apply to the SD configuration (Fig. 11), the main difference being the blob dynamics is better described by the CS scaling rather than by the RX one through a wider portion of the main SOL.

Globally, the blobs in the simulations are therefore in the regimes one would expect from the values of $\Lambda, \epsilon_{x}$ and $\Theta$, i.e. the CS and CI ones. The better agreement in the main SOL with the RX rather than the CS scaling could be due to the decrease in radial velocity associated to blob spinning [33], as it will be further discussed in section 6 . Local differences between the expected regime and the one better agreeing with the measured $v_{r}$ can be explained by non-local effects, e.g. the history of the blob traveling radially through zones associated with different regimes and needing time to adapt its velocity accordingly.

This comparison shows that filaments can have different dynamic regimes in the same configuration, changing from one to another in different regions of the SOL. They so potentially evolve and adapt their radial velocity as they travel radially outwards. This detail is not captured by the standard picture used to describe blob dynamics. Nevertheless, the theoretical model describing the radial propagation of a blob in a simplified geometry and background plasma describes reasonably well the different (averaged-) blob regimes also in these 3D simulations featuring a realistic magnetic 

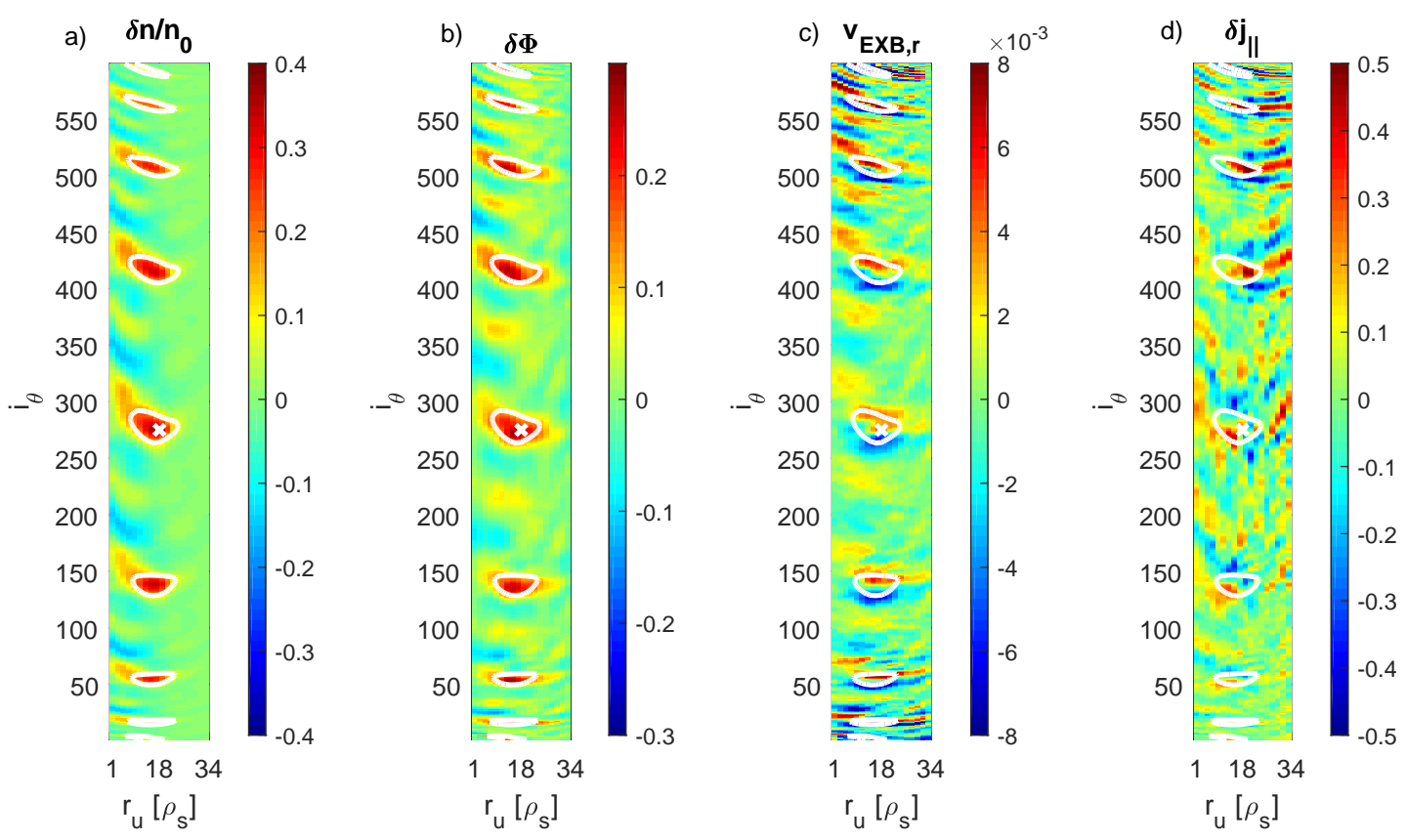

Figure 12: a) Density fluctuation $\delta n$, b) plasma potential fluctuation $\delta \Phi$, c) radial $\mathbf{E} \times \mathbf{B}$ velocity $v_{\mathbf{E} \times \mathbf{B}, r}$, and d) parallel current fluctuation $\delta j_{\|}$resulting from the 3D CAS in the LFS main SOL of the LSN configuration, for the reference point being at the OMP halfway between the two separatrices (white crosses). The contours $\delta n=\sigma$ are shown in white.

geometry and a turbulent background plasma. We remark indeed that despite the use of the CAS technique involving substantial averaging of the blob profiles, the scattering of the resulting data is still evident. The latter can be interpreted as an effect of the turbulent background plasma, resulting in mutual blob interactions, that can cause deviations from the average-blob model, suggesting even larger differences for the dynamics of single filaments. The latter point can not be investigated by means of CAS, and will be further discussed in the following section 7 .

\section{$6 \quad 3 D$ structure of blobs}

The three-dimensional structure of blobs in the OMP main SOL is described using the 3D CAS technique described in section 2.2. The resulting $\mathrm{CAS} \delta n, \delta \Phi, v_{\mathbf{E} \times \mathbf{B}, r}$ and $\delta j_{\|}$are shown on Fig. 12 in the LFS main SOL of the LSN configuration, for the reference point being at the OMP radially halfway between the two separatrices (white crosses). The contours $\delta n=\sigma$, defining approximately the blob cross section, are shown in white. The plots extend poloidally from the lower (main) $\mathrm{X}$-point (at the bottom of the plots) to the upper (secondary) one, at the top of the plots. As a result, the monopole in density fluctuation at the OMP (Fig. 12a) does not coincide with a dipole in the plasma potential fluctuations (Fig. 12b). Indeed, the average phase shift $\delta_{p h} \equiv$ $\arccos \left[\langle\delta n \delta \Phi\rangle /\left(\left\langle\delta n^{2}\right\rangle^{1 / 2}\left\langle\delta \Phi^{2}\right\rangle^{1 / 2}\right)\right]$ in the main SOL is approximately $\delta_{p h} \sim \pi / 4$ and $\delta_{p h} \sim \pi / 3$ for the LSN and SD configuration, respectively. This is consistent with previous turbulence simulations in limiter geometry $[23,34]$, and suggests that other instabilities such as drift waves $\left(\delta_{p h} \sim 0\right)$ may superimpose to the main interchange one $\left(\delta_{p h}=\pi / 2\right)$ [34].

The small phase shift in between $\delta n$ and $\delta \Phi$ is reflected in a more dipole-like structure for the CAS 


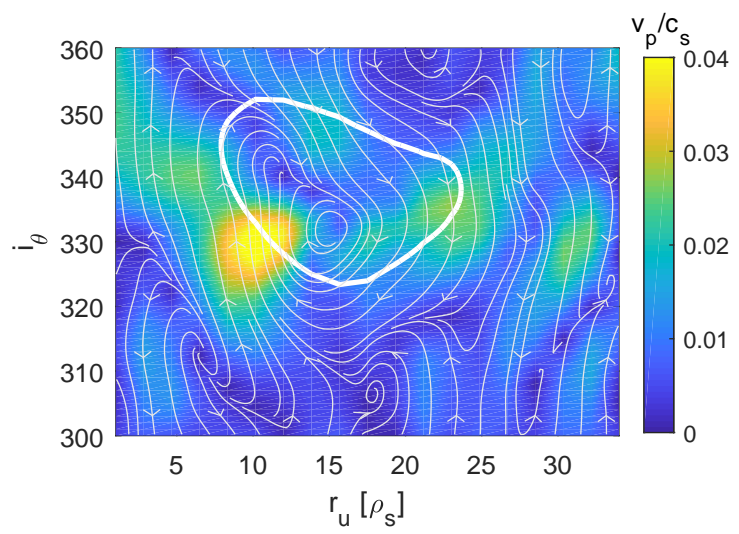

Figure 13: CAS fluctuating poloidal velocity $v_{p}$ together with the relative streamlines (white, arrows) for the average blob in Fig. 12 (thick white contour) at the OMP.

$v_{\mathbf{E} \times \mathbf{B}, r}$ (Fig. 12c). This questions the assumption made in section 4 , and more generally in the theoretical models for blob propagation, i.e. that the blob velocity coincides with the $\mathbf{E} \times \mathbf{B}$ velocity at the point of maximum density fluctuations. This can partly explain some of the differences between the predicted scalings and the CAS results as discussed in section 5. The CAS parallel current fluctuations $\delta j_{\|}$(Fig. 12d) exhibit an even more complex dipole-like pattern.

The dipolar structure of $v_{\mathbf{E} \times \mathbf{B}, r}$ leads to blob spinning, as it is shown in Fig. 13, where the CAS fluctuating poloidal velocity $v_{p}$ is shown together with the relative streamlines for the average blob in Fig. 12 at the OMP. Blob spinning can increase blob coherence and can impact both the radial and poloidal blob motion [33]. We remark that in Ref. [33] blob spinning arises of a natural consequence of the blob having an internal temperature profile; this is not the case for our isothermal $\left(T_{e}=T_{i}=1\right)$ simulations, and it has to be considered therefore to be directly caused by the drift wave-like potential monopole of our filaments.

In the following, we evaluate the connection of the blobs from the OMP to the target, both in terms of density and current fluctuations. Since the blobs are field aligned and, as discussed above, are assimilable to a density monopole in a poloidal plane, we evaluate the connection of the density fluctuations by studying the value of the CAS $\delta n$ along a field line passing through the reference point used for the CAS, i.e. the maximum of $\delta n$ at the OMP. The so evaluated relative fluctuations $\delta n / n$ are shown in Fig. 14 for different reference points at the OMP for the LSN (a) and SD (b) configurations. In the proximity of the primary separatrix, the density fluctuations at the OMP are disconnected from the LFS target, as $\delta n / n \leq 0$ from the X-point location up to the target. Further into the main SOL, the filaments are attached to the divertor plate, as $\delta n / n>0$ from the OMP to the LFS end of the field line. We remark that $\delta n / n$ substantially decreases when moving from the LFS to the HFS $\left(s / L_{\|}>0.6\right)$, according to the damping of turbulence due to unfavorable curvature for the the interchange instability.

As far as the electrical connection is concerned, the picture is more complicated. Indeed, the fluctuations of parallel current $\delta j_{\|}$along the field line passing through the maximum of the density fluctuation at the OMP also go to zero for the blobs close to the LCFS, as shown in Fig. 15a for the LSN configuration. Since $j_{\|}$exhibits a complex structure internally to the blob (as discussed above and shown in Fig. 12), focusing on the value of $\delta j_{\|}$along a single field line to evaluate the blob electrical connection to the divertor plates might not be the best choice. In Fig. 15b we plot instead the quantity $C_{j}(s)=\int_{S}\left|\delta j_{\|}\right| d S$, where the surface integral is evaluated on every poloidal cut by following all the field lines going through the $\delta n / \sigma=1$ contour at the OMP for each reference 

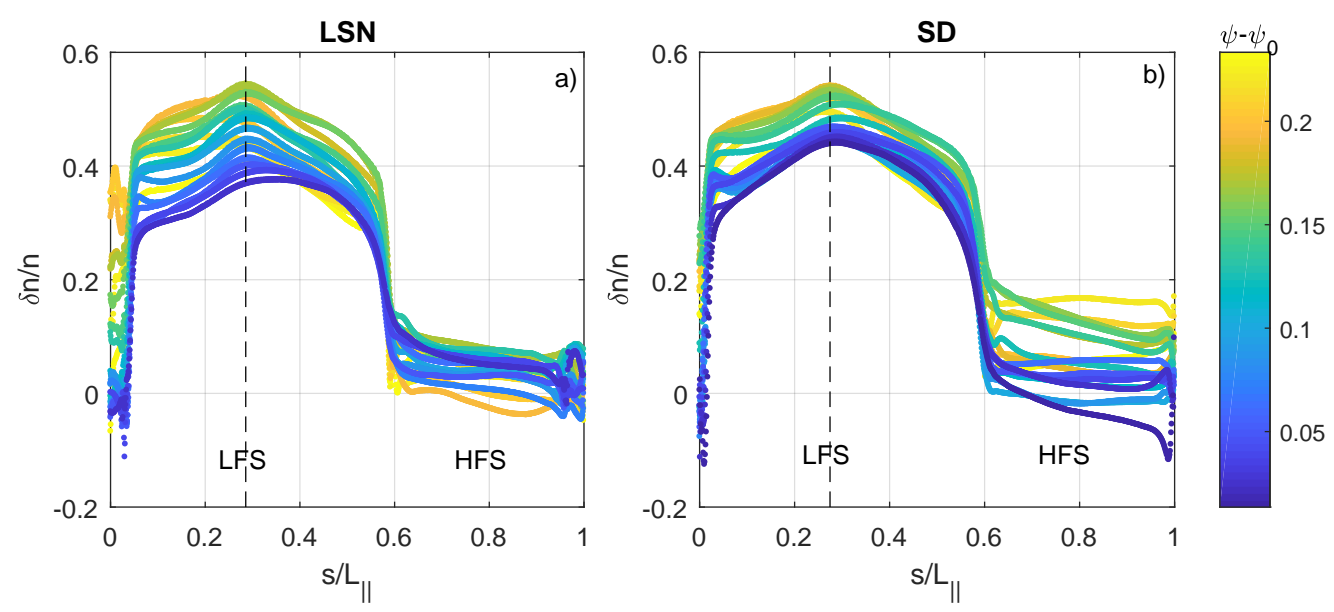

Figure 14: Relative density fluctuation $\delta n / n$ along the field line obtained from the 3D CAS for different reference points at the OMP, for the a) LSN and b) SD configuration. The normalized parallel coordinate $s / L_{\|}=0$ at the LFS target and $s / L_{\|}=1$ at the HFS target; the position of the OMP is shown with a black dashed line. The color coding is the poloidal flux of the reference point.
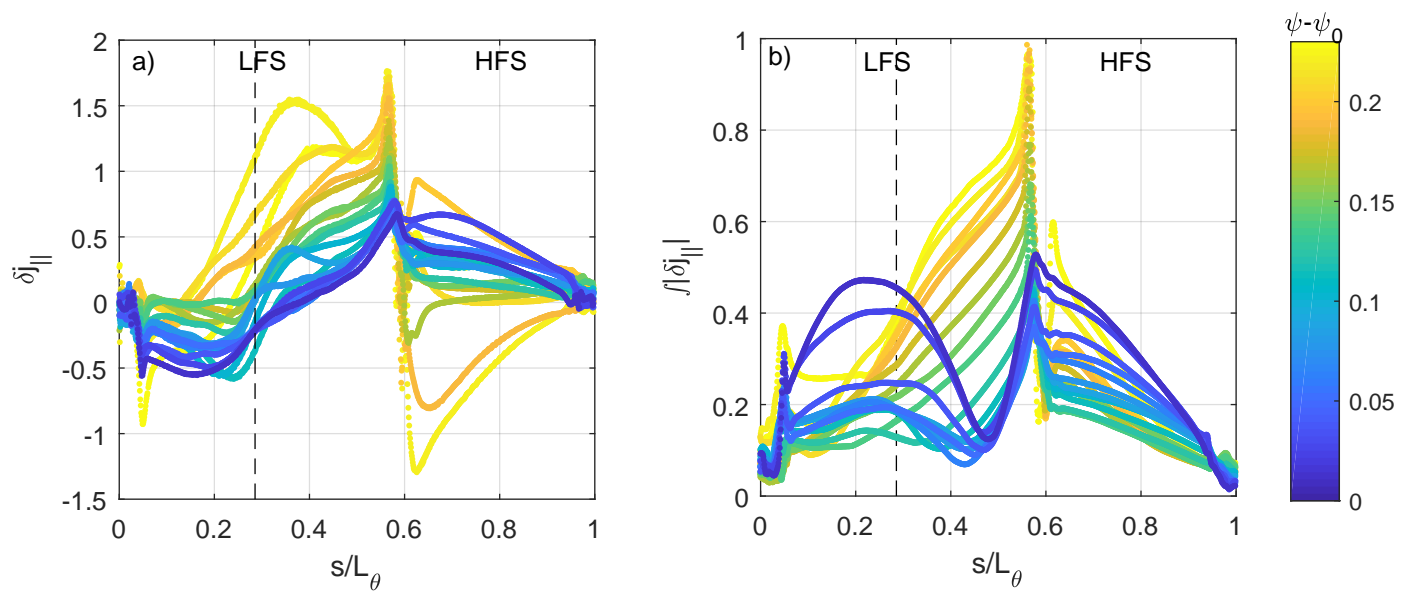

Figure 15: a) Parallel current density fluctuation $\delta j_{\|}$along the field line obtained from the 3D CAS for different reference points at the OMP for the LSN configuration. b) Surface integrated current $C_{j}(s)$ (definition in the text) along the field lines. The normalized parallel coordinate $s / L_{\|}=0$ at the LFS target and $s / L_{\|}=1$ at the HFS target; the position of the OMP is shown with a black dashed line. The color coding is the poloidal flux of the reference point.

blob. As a result, for the blobs close to the LCFS the current $C_{j}(s)$ integrated over the surface is substantially reduced when going from the OMP through the LFS X-point, but it is never null. Similar conclusions hold for the SD configuration.

\section{3D structure and dynamics of single blobs}

The BRAT algorithm (described in section 2.3) is applied on the simulations in both LSN and SD configurations, resulting in the detection of 2273 and 1850 blobs, respectively. Among the detected 
blobs, $53.4 \%$ (61.5\%) is first detected (or "naturally born") in the LSN (resp. SD) configuration, while $20.2 \%(16.7 \%)$ is born from splitting events, $19.2 \%$ (17.1\%) from merging events, and $7.1 \%$ (4.8\%) results from combined merging and splitting events. This result, i.e. approximately half of the tracked blob being involved in mutual interactions, is consistent with previous $2 \mathrm{D}$ tracking analysis on full turbulence simulations of limited plasmas performed with a different code[16]. This highlights the impact of a consistent turbulent plasma background on blob dynamics, which is not included in seeded blob simulations.

We can categorize the detected filaments into different populations in the LSN (SD) configuration. Indeed, $13.2 \%(15.7 \%)$ of the blobs are contained inside the LCFS, $39.7 \%(47.6 \%)$ are entirely located in the HFS SOL, while $37.8 \%(32.4 \%)$ are entirely located in the LFS SOL. Moreover, $8.0 \%$ $(3.3 \%)$ of the blobs extend into both the HFS and the LFS, while $14.1 \%(0 \%)$ are entirely contained in the divertor (zones 1,2,3,4,7 and 8 in Fig. 1 for the LSN, not present for the SD). In the latter category, $51.6 \%$ of the blobs are connected to the inner strike point and $37.6 \%$ are connected to the outer strike point, while the remaining $10.8 \%$ are in the private flux region. The divertor blobs, being entirely located below the X-point and therefore disconnected from the OMP, are most probably generated at this location by turbulence, consistently with previous TOKAM3X investigations [35]. The dynamics of this kind of blobs, also observed experimentally in Ref. [31,36], is not described by the theoretical models (e.g. [5]) that are developed for blobs located at the OMP, and thus their radial velocity could scale differently. The investigation of this kind of blobs and their impact on divertor heat loads is therefore advised and will be carried on in future works.

The detected blobs survive on average $687(695) \tau_{c}$ in the LSN (SD) configuration, the most probable value being $300 \tau_{c}$ (corresponding to three sampling steps, being the output frequency $10^{-2} \tau_{c}^{-1}$, used as a cutoff in order to compute the velocity), with a probability distribution approximately exponentially decaying for all the blob populations, as is shown for the LSN configuration in Fig. 16a. Statistically speaking the detected blobs are also spatially short, with the mean toroidal extension $\langle\Delta \phi / 2 \pi\rangle=0.89$ (0.81) turns, and the most probable value is close to 0. Most of the short blobs though are located entirely at the HFS or entirely at the LFS, mostly outside the secondary separa-
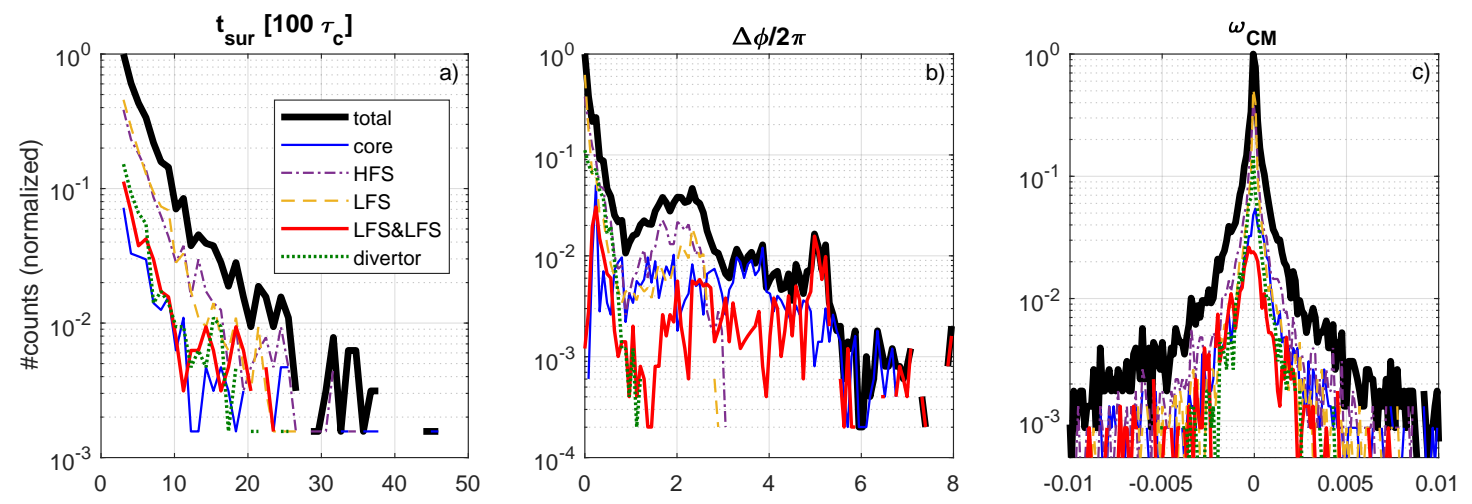

Figure 16: Histogram of a) blob survival time $t_{\text {sur }}$ b) toroidal extension $\Delta \phi / \pi$ c) toroidal angular frequency $\omega_{C M}$ for the LSN configuration (black), with the contributions from blobs detected inside the LCFS (solid blue), blobs located entirely at the HFS (dash-dotted violet), entirely at the LFS (dashed yellow), shared between LFS and HFS (solid red), and located entirely in the divertor (dotted green). 

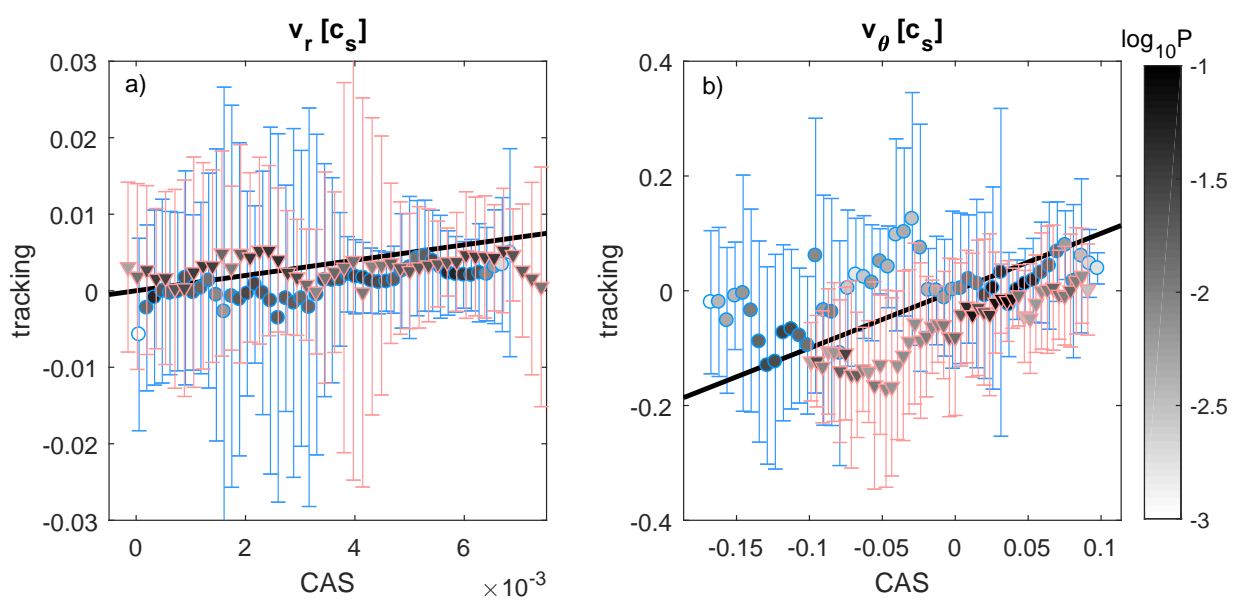

Figure 17: Comparison of the a) radial and b) poloidal components of blob velocities resulting from the tracking $v_{t r}$ with the CAS ones $v_{C A S}$ for the LSN (circles, blue errorbars) and SD (triangles, red errorbars) configurations, color coded with the occurrence of $v_{C A S}$. The errorbars represent the standard deviation of the measured $v_{t r}$. The equality line $v_{t r}=v_{C A S}$ is plotted in black.

trix or in the private flux regions, i.e. in zones where the background density is small $\bar{n}<n_{0} / 100$, that have been neglected in the previous CAS analysis. Conversely, the blobs that extend from the LFS to the HFS are winded on average on 2.59 (3.34) toroidal turns, with a probability distribution peaking at 5 (4.5) turns (Fig. 16b), this value corresponding to a full poloidal turn around of the plasma, $q_{95}$ being around 4 and increasing in the SOL. On the contrary, the divertor blobs are small $(\Delta \phi / 2 \pi \lesssim 1)$, consistently with their limited poloidal extension by definition. We remark that the filaments are on average shorter for the SD configuration, which is consistent with the field lines being shorter in this configuration due to the removal of the region below the X-point. The probability distribution of filament angular velocity $\omega_{C M}$ is peaked around 0 , as shown in Fig. 16c, and slightly asymmetric, with $\left\langle\omega_{C M}\right\rangle=-1.3 \cdot 10^{-4} \tau_{c}^{-1}\left(-1.0 \cdot 10^{-4} \tau_{c}^{-1}\right)$. For the OMP blobs the asymmetry increases, and the average $\omega_{C M}$ is $-5.7 \cdot 10^{-4} \tau_{c}^{-1}$ for the LSN configuration, and $-7.5 \cdot 10^{-5} \tau_{c}^{-1}$ for the SD configuration. This is consistent with the average negative toroidal velocity resulting from the CAS analysis presented in section 4, and with its reduction when moving from the LSN configuration to the SD one.

In the following we compare the results from BRAT to the ones from the CAS, exposed in section 4. As the blob poloidal size $a_{\theta}$ is computed with analogous procedures in both cases, the BRAT $a_{\theta}$ are on average directly proportional to the CAS ones, but of course with a huge spread due to the fact that the BRAT values correspond to single blobs while the CAS ones are averaged values. The same conclusion holds for the relative density fluctuations $\delta n / n$, with the difference that the CAS values are here computed as a volume average for each single blob toroidal slice, and the CAS values are the (averaged) peak values. On average the linear relation holds $(\delta n / n)_{B R A T}=A(\delta n / n)_{C A S}$, with $A \sim 0.6$ and 0.5 for the LSN and SD case, respectively. One more meaningful comparison is the one between the velocities resulting from the two techniques, determined in substantially different ways. Indeed, while the CAS velocities rely on the assumption that the blob velocity corresponds to the $\mathbf{E} \times \mathbf{B}$ drift at the blob location, the BRAT velocities result directly from the tracking in time of the three-dimensional structures, and no assumption on their nature is made a priori. This comparison is shown in Fig. 17, where we compare the velocities computed from the tracking algorithm, $v_{t r}$, with the ones resulting from the CAS, $v_{C A S}$. This analysis is carried on an angular sector centered 


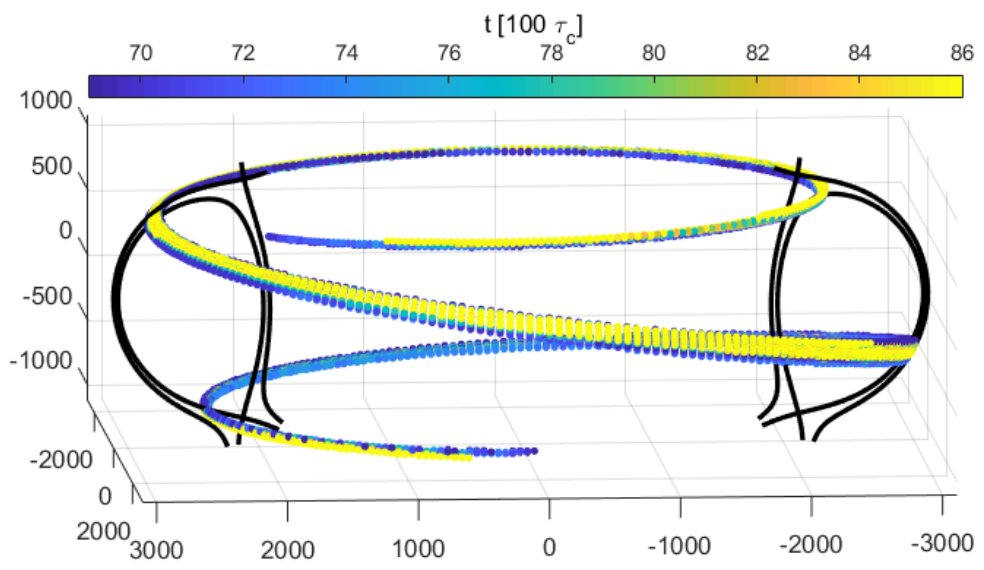

Figure 18: Three-dimensional structure of a detected blob evolving in time (color coding is time).
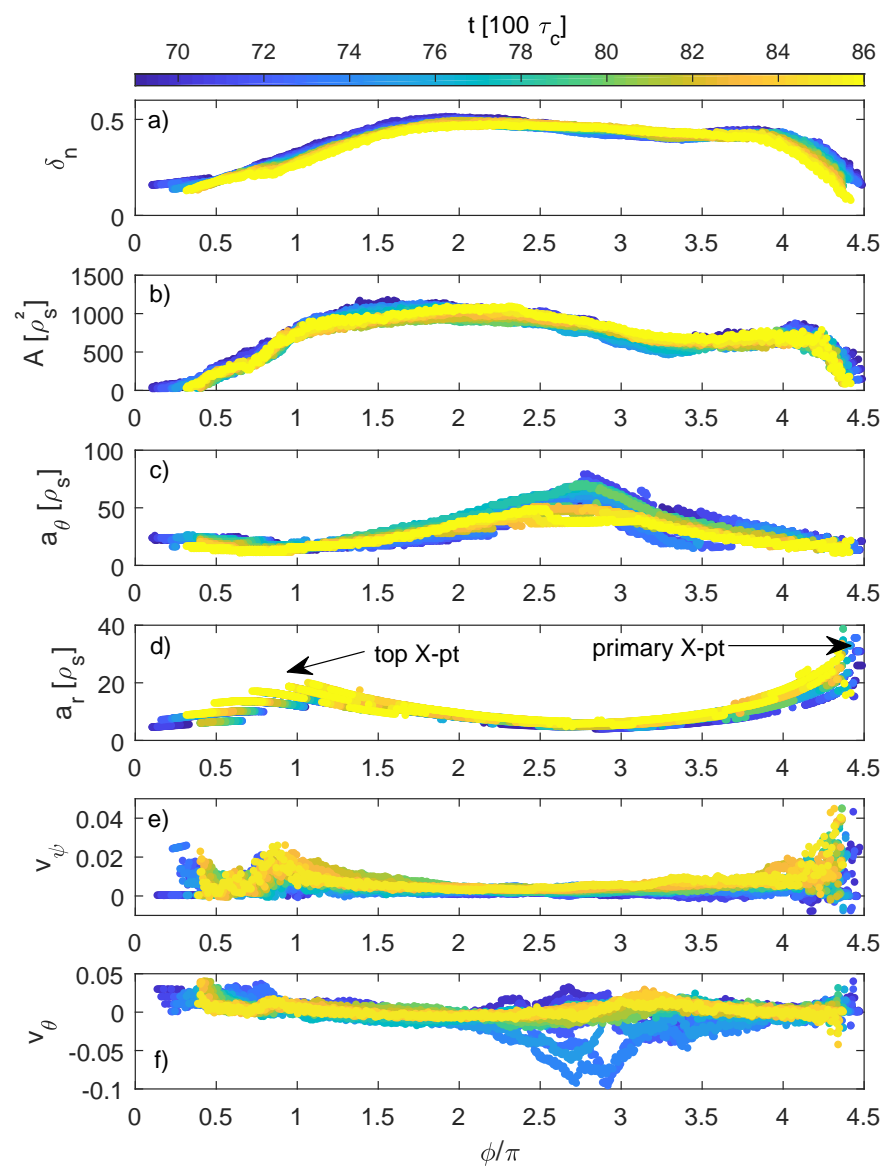

Figure 19: Evolution in time of the toroidal profiles of a) blob density fluctuation $\delta n$, b) area in the poloidal cross section $A, \mathrm{c}$ ) poloidal size $a_{\theta}$ and d) radial size $a_{r}$, e) radial velocity $v_{\psi}$ and f) poloidal velocity $v_{\theta}$ resulting from the tracking. Color coding is time. 

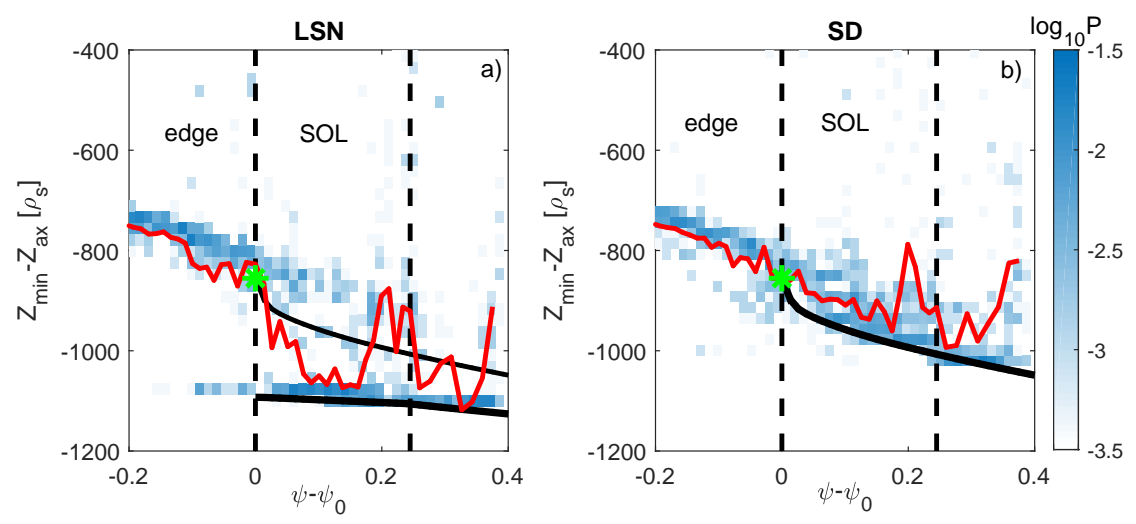

Figure 20: Lower point reached by the blobs connected to the OMP $Z_{a x}-Z_{\min }$, plotted as a function of $\psi-\psi_{0}$ (probability distribution in blue, average value in red), for the LSN (a) and SD (b) configurations. The position of the X-point is shown with a green star, and the vertical position of the line passing through the $\mathrm{X}$-point and perpendicular to the flux surfaces is shown with a black line. The divertor plate is plotted with a thick black line. The position of the two separatrixes is shown with dashed lines.

around the midplane, and $v_{C A S}$ have been binned for an easier comparison, the occurrence of each binned value being given in the picture by the color coding. The average $v_{t r}$ for blobs crossing the corresponding CAS points and its standard deviation have been computed for both the LSN (circles, blue errorbars) and the SD configuration (triangles, red errorbars). As a result, we notice how the velocities resulting from the tracking are in average consistent with $v_{C A S}$ (within errorbars, the equality line being plotted in black), for both the radial and poloidal component; though, the scattering is usually huge, even though it is reduced for the $v_{C A S}$ with higher occurrence. We also remark that, while on average the radial component of $v_{t r}$ is positive, the number of blobs propagating radially inwards is non-negligible. Radial negative velocities could result from nonlinear effects such as blob rotation, interaction between multiple blobs or the splitting of a single one. Such velocities have been already observed both in experiments $[13,37]$ and simulations with other codes $[16,17]$.

The three-dimensional filamentary structures can indeed evolve in time in complex ways. Even though exposing a zoology of blob trajectories is tempting, it would be unnecessarily long and not relevant in statistical terms. Therefore, we show here just one example in Fig. 18, were we plot the time evolution of a blob extending in the LFS SOL approximately from the secondary X-point to the primary one. The corresponding evolution of the toroidal profiles of the blob density fluctuation $\delta n$, the area in the poloidal cross section $A$, the poloidal and radial size $a_{\theta}$ and $a_{r}$, and the radial and poloidal velocities resulting from the tracking $v_{\psi}$ and $v_{\theta}$ are shown in Fig .19. In this figure, the secondary X-point (top of the plasma) is on the left, while the main X-point (bottom of the plasma) is on the right. We can see that, as the blob approaches the main X-point, $\delta n$ is decreased and the blob squeezes ( $A$ is also decreased); more specifically, the blob is squeezed in the poloidal direction ( $a_{\theta}$ decreases) while it elongates in the radial direction ( $a_{r}$ increases) according to the deformation of the flux tube. For the blob to remain field aligned in its motion, $v_{\psi}$ is also increased at the X-point. $v_{\theta}$, mainly negative at the OMP (blob drifting upwards), has a complex evolution both in time and space.

The squeezing of the blobs in the toroidal direction at the X-point, at scales comparable to the gyro-radius (or, in this case, to the resolution of the simulation) where collisional/diffusive processes start to be important as suggested in Ref. [31], is the most probable cause of their disconnection from 

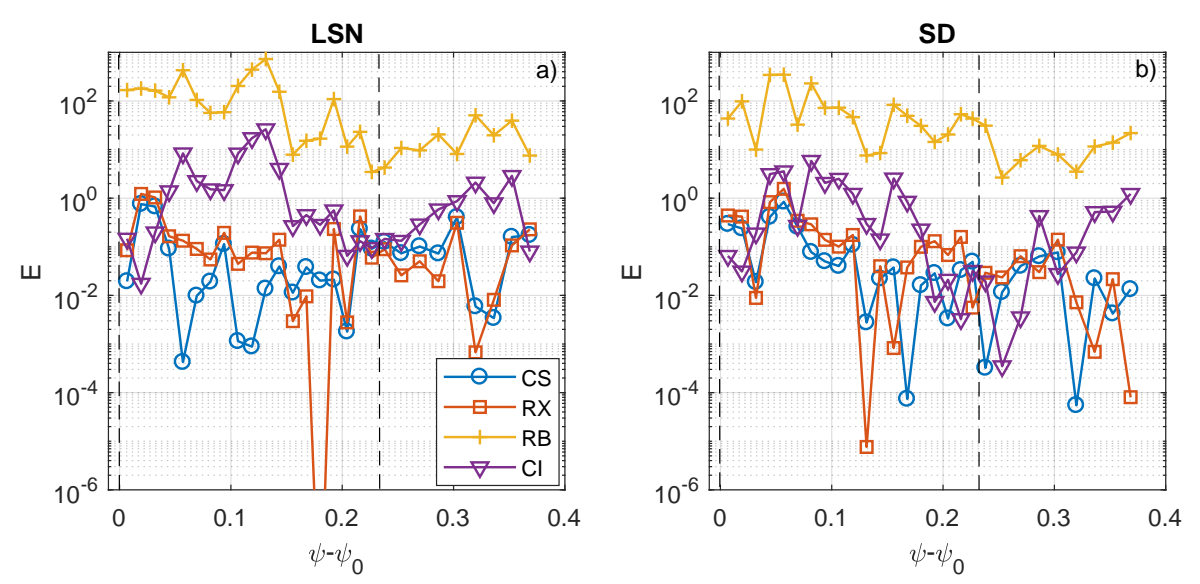

Figure 21: Relative error $E=\left(\left\langle\tilde{v}_{t r}\right\rangle-v_{s c}\right)^{2} / \sigma_{v, t r}^{2}$ between the radial velocities measured resulting from BRAT (binned) and the predicted values from the different scalings from Refs. [5, 20], for the a) LSN and b) SD configurations.

the target plates. Indeed, in Fig. 20, we consider the lower point reached by the blobs connected to the OMP, plotted as a function of $\psi-\psi_{0}$ (probability distribution in blue, average value in red). For the LSN case, close to the separatrix the blobs do not extend further than the X-point (green star, black line) and do not reach the divertor plate (thick black line). Further in the SOL, the blobs extend up to the divertor plate, and the OMP and target plate are therefore connected. We remark that, even though cases of a single blob starting close to the LCFS disconnected from the divertor plate and reconnecting to it as it moves radially outwards in the SOL are observed here, they are statistically not relevant as the filaments motion is primarily in the poloidal direction. Furthermore, the blobs are also disconnected from the target close to the secondary separatrix, but here it is more probably due to the strong shear in poloidal velocity described in sections 3 and 4 , rather than to the squeezing of the filamentary structure as described here for the main X-point. For the SD configuration, where the X-point coincides with the target plates, the blobs still seem not to reach the divertor plate close to the separatrix, while they are connected to it further in the SOL. We remark that, in addition to the squeezing of the filaments, magnetic shear could play a role in the loss of coherence of blobs at the X-point, as shown in seeded blob simulations [14].

Due to the huge scattering in the tracked radial velocities, including also negative values, which are not taken into account by the theoretical models, the comparison with the scalings discussed in section 5 becomes more difficult in the tracking case. To reduce the scattering in measured velocities, we binned the results on the value of $\psi$, resulting in the average values $\left\langle\tilde{v}_{t r}\right\rangle(\psi),\left\langle\tilde{a}_{t r}\right\rangle(\psi)$, $\left\langle\frac{\delta n}{n}\right\rangle(\psi)$, where the brackets denotes binning over $\psi$. Using these values, we can compute the predicted velocities $v_{s c}$ for the CS, RX, RB and CI scaling respectively. In Fig. 21, we quantify the goodness of the prediction through the relative error $E=\left(\left\langle\tilde{v}_{t r}\right\rangle-v_{s c}\right)^{2} / \sigma_{v, t r}^{2}$, where $\sigma_{v, t r}$ is the standard deviation of the binned radial velocity $\left\langle\tilde{v}_{t r}\right\rangle$. As a result, for both the LSN (a) and SD configuration (b), the CS or RX scaling agree best with the measured velocities through all the SOL, except in the vicinity of the two separatrixes, where the the CI scaling provides a better (or at least comparably good) prediction. The RB scaling overestimates the blob radial velocity through all the SOL. 


\section{Conclusion}

The effect of X-points and of the divertor configuration on turbulence and more specifically on the blobs dynamics is studied in this paper, using isothermal simulations of the WEST tokamak performed with the fluid code TOKAM3X. Two magnetic topologies are compared, namely the standard Lower Single Null (LSN) and the Shallow Divertor (SD), the second one featuring the primary X-point directly in contact with the lower divertor plate. Both configurations show the activation of secondary strike points at the top of the plasma through the secondary X-point. While in the LSN configuration the divertor particle flux profiles are well described by the usual convolution of an exponential decay and a gaussian taking into account diffusive processes in the private flux region, the SD case exhibits almost gaussian divertor particle flux profiles, and diffusion dominates. To characterize the effect of the magnetic geometry on turbulence, we investigate blobs dynamics. We first apply a Conditional Average Sampling (CAS) technique, and we compare the resulting blob sizes and velocities in a surrounding of the outer midplane (OMP) with the theoretical scalings in Refs. [5, 20]. As a result, this theoretical model describing the radial propagation of a blob in a simplified geometry and background plasma describes reasonably well the different blob regimes observed in the 3D simulations featuring a realistic magnetic geometry and a turbulent background plasma. The filaments are observed to follow different scalings depending on the SOL region; in particular, they agree with the sheath connected scaling or the resistive X-point one (giving similar predictions for the considered cases) in the main SOL, except close to the separatrix, where the filament dynamics agree better with the connected ideal-interchange scaling, where the instability drive is balanced by fanning-enhanced inertia close to the X-point. We complement these results with a 3D CAS analysis, showing how the averaged filaments at the OMP are disconnected from the target when close to the separatrix, being the density fluctuation along the field line $\delta n \leq 0$ below the X-point. A similar investigation on the filament electrical connection to the target gives no clear result, mainly due to the difficulty of clearly defining a criterion for the electrical connection of blobs, exhibiting internal complex current dipole-like structure. Qualitatively, the same results hold for both the LSN and SD cases.

A novel 3D blob recognition and tracking algorithm has been implemented and presented here. Its application to the simulation results provides the evolution in time of the three-dimensional blob structures, and of the toroidal profiles of the associated density fluctuation, poloidal and radial sizes and velocities. The resulting velocities at the OMP agree on average with the ones resulting from the CAS analysis, despite a huge scattering in values. A non negligible fraction of blobs exhibits negative radial velocities, not described by the usual theoretical models, still experimentally observed. These results evidence how a turbulent background plasma with mutual interactions in between filaments can alter their dynamics, and how the trajectory of a single filament can substantially differ from the average-blob evolution (CAS) and from the simplified theoretical prediction. The comparison of the velocities and sizes resulting from the tracking with the theoretical scalings recovers qualitatively the results from the CAS analysis, while the investigation of the poloidal extent of the blobs connected to the OMP agrees with the results from the 3D CAS, showing how the blobs in correspondence of the separatrixes do not reach the target plates. Following the evolution of the 3D filaments structure, we see how the filaments squeeze in the poloidal direction while stretching in the radial one in correspondence of the X-point, increasing at the same time their radial velocity. The poloidal squeezing up to scales comparable to the ion gyroradius or where in any case collisional/diffusive processes become dominant is probably the main cause of the blob disconnection from the target, as suggested in Ref. [31]. Furthermore, in the LSN case a fraction of the blobs are generated by turbulence below the X-point. The dynamics of this kind of blob, not described by the usual theoretical models, need to be investigated in future works in order to asses their impact on divertor 
heat loads.

As a final picture, in these simulations the combination of high flux expansion and magnetic shear disconnects the blobs from the divertor plates in the vicinity of the separatrix. This results, for the LSN case, in a quiescent zone below the X-point, as previously observed in both experiments $[31,36]$ and simulations [30], though active turbulence along the divertor leg creates new filaments in this region. In the SD case, where the X-point is directly in contact with the divertor plate, the disconnection of the filaments from the target (over a wider region due to the high X-point flux expansion), with no further possibility of newly born filaments below the X-point, results in more gaussian-like particle flux profiles on the target plates.

\section{Acknowledgments}

This work was granted access to the HPC resources of CINES, under the allocations A0030506912 \& A0050506912 made by GENCI, to the HPC resources of Aix-Marseille University financed by the project Equip@Meso (ANR-10-EQPX-29-01), and to the EUROfusion High Performance Computer (Marconi-Fusion) under the project HEAT. The project leading to this publication (TOP project) has received funding from Excellence Initiative of Aix-marseille University- A*MIDEX, a french "Investissements d'Avenir" programme. This work has been carried out within the framework of the EUROfusion Consortium and has received funding from the Euratom research and training programme 2014-2018 and 2019-2020 under grant agreement No 633053 (project WP17-ENR-CEA08). The views and opinions expressed herein do not necessarily reflect those of the European Commission.

\section{References}

[1] P.H. Diamond et al. Vorticity dynamics, drift wave turbulence, and zonal flows: a look back and a look ahead. Plasma Physics and Controlled Fusion, 53:124001, 2011.

[2] S.I. Krasheninnikov et al. Recent theoretical progress in understanding coherent structures in edge and SOL turbulence. Journal of Plasma Physics, 74:679-717, 2008.

[3] G. Birkenmeier et al. Filament transport, warm ions and erosion in ASDEX Upgrade L-modes. Nuclear Fusion, 55:033018, 2015.

[4] Y. Sarazin and Ph. Ghendrih. Intermittent particle transport in two-dimensional edge turbulence. Physics of Plasmas, 5:4214, 1998.

[5] J. R. Myra et al. Collisionality and magnetic geometry effects on tokamak turbulent transport. I. A two-region model with application to blobs. Physics of Plasmas, 13:112502, 2006.

[6] P. Manz et al. Filament velocity scaling laws for warm ions. Phisics of Plasmas, 20:102307, 2013.

[7] L. Easy et al. Investigation of the effect of resistivity on scrape off layer filaments using three-dimensional simulations. Phisics of Plasmas, 23:102512, 2016.

[8] S.H. Mueller et al. Probabilistic analysis of turbulent structures from two-dimensional plasma imaging. Physics of Plasmas, 13:100701, 2006. 
[9] D. Carallero et al. Experimental validation of a filament transport model in turbulent magnetized plasmas. Physical Review Letters, 115:215002, 2015.

[10] A. Kirk et al. L-mode filament characteristics on MAST as a function of plasma current measured using visible imaging. Plasma Physics and Controlled Fusion, 58:085008, 2016 .

[11] N. Vianello et al. Modification of SOL profiles and fluctuations with line-average density and divertor flux expansion in TCV. Nuclear Fusion, 57:116014, 2017.

[12] N. Fedorczak et al. On the dynamics of blobs in scrape-off layer plasma: model validation from two-dimensional simulations and experiments in Tore Supra. Contributions to Plasma Physics, pages 1-7, 2018.

[13] C.K. Tsui et al. Filamentary velocity scaling validation in the TCV tokamak. Physics of Plasmas, 25:072506, 2018.

[14] W.A. Gracias et al. The impact of magnetic shear on the dynamics of a seeded 3D filament in slab geometry. Nuclear Materials and Energy, 12:798807, 2017.

[15] D. Schworer et al. Influence of plasma background on 3D scrape-off layer filaments. Plasma Physics and Controlled Fusion, 61:025008, 2019.

[16] F. Nespoli et al. Blob properties in full-turbulence simulations of the TCV scrape-off layer. Plasma Physics and Controlled Fusion, 59:055009, 2017.

[17] M. Churchill et al. Pedestal and edge electrostatic turbulence characteristics from an XGC1 gyrokinetic simulation. Plasma Physics and Controlled Fusion, 59:105014, 2017.

[18] P. Paruta et al. Blob velocity scaling in diverted tokamaks: A comparison between theory and simulation. Physics of Plasmas, 26:032302, 2019.

[19] P. Tamain et al. The TOKAM3X code for edge turbulence fluid simulations oftokamak plasmas in versatile magnetic geometries. Journal of Computational Physics, 321:606-623, 2016.

[20] J. R. Myra et al. Blob birth and transport in the tokamak edge plasma: Analysis of imaging data. Physics of Plasmas, 13:092509, 2006.

[21] C. Bourdelle et al. WEST Physics Basis. Nuclear Fusion, 55:063017, 2015.

[22] F. Riva et al. Blob dynamics in the TORPEX experiment: a multi-code validation . Plasma Physics and Controlled Fusion, 58:044005, 2016.

[23] C. Baudoin et al. Turbulent heat transport in TOKAM3X edge plasma simulations. Contributions to Plasma Physics, 58:484-489, 2018.

[24] P. Manz et al. Plasma blob generation due to cooperative elliptic stability. Physical Review Letters, 107:195004, 2011.

[25] C. Theiler et al. Blob motion and control in simple magnetized plasmas. Physics of Plasmas, 18:055901, 2011. 
[26] S.J. Zweben et al. Edge and SOL turbulence and blob variations over a large database in NSTX. Nuclear Fusion, 55:093035, 2015.

[27] B. Nold. Investigation of turbulent structures in the edge of magnetized plasmas. PhD thesis Universitat Stuttgart, http://dx.doi.org/10.18419/opus-2024, 2012.

[28] F. Wagner. A study of the perpendicular particle transport properties in the scrapeoff layer of ASDEX. Nuclear Fusion, 5:525, 1985.

[29] T. Eich et al. Inter-ELM power decay length for JET and ASDEX Upgrade: measurement and comparison with the heuristic drift-based model. Physical Review Letters, 107:215001, 2011.

[30] D. Galassi et al. Drive of parallel flows by turbulence and largescale E B transverse transport in divertor geometry. Nuclear Fusion, 57:036029, 2017.

[31] N.R. Walkden et al. Quiescence near the X-point of MAST measured by high speed visible imaging. Nuclear Fusion, 57:126028, 2017.

[32] D.A. D'Ippolito et al. Blob Transport in the Tokamak Scrape-off-Layer. Contributions to Plasma Physics, 44:205-216, 2004.

[33] J. R. Myra et al. Convective transport in the scrape-off-layer by nonthermalized spinning blobs. Physics of Plasmas, 11:4267, 2004.

[34] J. Loizu et al. Effect of the limiter position on the scrape-off layer width, radial electric field and intrinsic flows. Nuclear Fusion, 54, 2014.

[35] A. Gallo, N. Fedorczak, S. Elmore, R. Maurizio, H. Reimerdes, C. Theiler, C. K. Tsui, J. A. Boedo, M. Faitsch, H. Bufferand, et al. Impact of the plasma geometry on divertor power exhaust: experimental evidence from TCV and simulations with SolEdge2D and TOKAM3X. Plasma Physics and Controlled Fusion, 60:014007, 2018.

[36] F. Scotti et al. Divertor leg filaments in NSTX-U. Nuclear Fusion, 58:126028, 2018.

[37] S. Zweben et al. Blob structure and motion in the edge and SOL of NSTX. Plasma Physics and Controlled Fusion, 58:044007, 2016. 